\title{
SLOW EIGENVALUES OF SELF-SIMILAR SOLUTIONS OF THE DAFERMOS REGULARIZATION OF A SYSTEM OF CONSERVATION LAWS: AN ANALYTIC APPROACH
}

\author{
XIAO-BIAO LIN
}

\begin{abstract}
The Dafermos regularization of a system of $n$ hyperbolic conservation laws in one space dimension has, near a Riemann solution consisting of $n$ Lax shock waves, a self-similar solution $u=u_{\epsilon}(X / T)$. In [19] it is shown that the linearized Dafermos operator at such a solution may have two kinds of eigenvalues: fast eigenvalues of order $1 / \epsilon$ and slow eigenvalues of order one. The fast eigenvalues represent motion in an initial time layer, where near the shock waves solutions quickly converge to traveling-wave-like motion. The slow eigenvalues represent motion after the initial time layer, where motion between the shock waves is dominant.

In this paper we use tools from dynamical systems and singular perturbation theory to study the slow eigenvalues. We show how to construct asymptotic expansions of eigenvalue-eigenfunction pairs to any order in $\epsilon$. We also prove the existence of true eigenvalue-eigenfunction pairs near the asymptotic expansions.
\end{abstract}

\section{INTRODUCTION}

The Dafermos regularization $[6,35,36,37]$

$$
u_{T}+f(u)_{X}=\epsilon T u_{X X}
$$

is a diffusively perturbed system of conservation laws in one space dimension. It has many similarity solutions of the form $u=u_{\epsilon}(X / T)$. This property is shared by the unperturbed system of conservation laws

$$
u_{T}+f(u)_{X}=0,
$$

but not by the usual viscous regularization

$$
u_{T}+f(u)_{X}=\epsilon u_{X X} .
$$

Using the change of variables

$$
x=X / T, \quad t=\ln T,
$$

the Dafermos regularization (1.1) becomes

$$
u_{t}+(D f(u)-x I) u_{x}=\epsilon u_{x x} .
$$

Date: March 15, 2005.

1991 Mathematics Subject Classification. 34E05 35L65 35L67 35P99.

Key words and phrases. conservation law, Riemann solution, Dafermos regularization, stability, eigenvalue, eigenfunction, SLEP method, asymptotic expansion.

Partially supported by the National Science Foundation under grant DMS-0406016. 
The same change of variables brings the system of conservation laws (1.2) to

$$
u_{t}+(D f(u)-x I) u_{x}=0 .
$$

In the new variables, the Dafermos regularization (1.4) appears to be a natural regularization of (1.5). It has been used to compute numerically one-parameter families of Riemann solutions [29].

A Riemann problem is an initial value problem for (1.2) with piecewise constant initial data

$$
u(X, 0)= \begin{cases}u^{\ell} & \text { if } X<0 \\ u^{r} & \text { if } X>0 .\end{cases}
$$

We consider a solution of the Riemann problem (Riemann solution) consisting of $n$ Lax shock waves with speeds $\bar{s}^{i}, i=1, \ldots, n$. Let $\bar{s}^{0}=-\infty$ and $\bar{s}^{n+1}=\infty$. Then

$$
u(X, T)=\bar{u}^{i} \quad \text { if } \bar{s}^{i}<X / T<\bar{s}^{i+1} .
$$

The stability of solutions of (1.3) near such a Riemann solution, with small jumps in $\bar{u}^{i}$, is considered in [20].

We shall not assume that shock waves are weak; instead we shall assume that the shock waves of the Riemann solution satisfy the viscous profile criterion. In other words, corresponding to the $i$ th shock wave there is a traveling wave solution $u(X, T)=q^{i}(\xi), \xi=\left(X-\bar{s}^{i} T\right) / \epsilon$, of (1.3). The function $q^{i}$ satisfies the traveling wave ODE

$$
(D f(u)-s I) u_{\xi}=u_{\xi \xi}
$$

with wave speed $s=\bar{s}^{i}$, and connects $\bar{u}^{i-1}$ to $\bar{u}^{i}$.

In $x$-coordinates, the Riemann solution becomes a piecewise constant, stationary solution $u_{0}(x)$ of $(1.5)$ :

$$
u_{0}(x)=\bar{u}^{i} \quad \text { for } \bar{s}^{i}<x<\bar{s}^{i+1} .
$$

Using geometric singular perturbation theory [12], Szmolyan proved that near a structurally stable Riemann solution $u_{0}(x)$ of (1.5) that consists of $n$ Lax shock waves and rarefactions, not necessarily weak, there are, for sufficiently small $\epsilon>$ 0 , stationary solutions $u_{\epsilon}(x)$ of (1.4) [34]. Szmolyan's work has been extended to other Riemann solutions [28, 22]. We will call stationary solutions of (1.4) RiemannDafermos solutions.

For the Riemann solutions we consider, which have $n$ Lax shock waves, the corresponding Riemann-Dafermos solutions have $n$ sharp internal layers near $x=\bar{s}^{i}, i=$ $1, \ldots, n$. In fact, $u_{0}(x)$ is the zeroth order expansion of $u_{\epsilon}(x)$ in regular layers. Using the stretched variable $\xi=\left(x-\bar{s}^{i}\right) / \epsilon, q^{i}(\xi)$ is the zeroth order expansion of $u_{\epsilon}(x)$ in the $i$ th singular layer.

Because $u_{\epsilon}(x)$ is a stationary solution, we wish to determine its stability by studying eigenvalues of the linearization of (1.4) at $u_{\epsilon}(x)$. It is known that:

(1) The initial value problem is well-posed for any initial data near $u_{\epsilon}(x)$ that approaches constants exponentially as $x \rightarrow \pm \infty$ [19].

(2) In the space of functions of order $e^{-\alpha|x|}$, the essential spectrum of the linearization lies to the left of the line $\operatorname{Re} \lambda \leq-\delta$ for some $\delta>0$ [19]. 
(3) The linearization is sectorial in the space of functions of order $e^{-\alpha|x|^{2}}[17]$. Thus, from the standard theory of analytic semigroups [8, 11, 24], in such spaces linearized stability of the Riemann-Dafermos solution is determined by the eigenvalues of the linearization.

(4) If the Riemann-Dafermos solution is linearly stable, nonlinear stability can be proved by a contraction mapping argument as in $[7,11]$.

It is known that there are both fast eigenvalues, of order $1 / \epsilon$, and slow eigenvalues, of order one. This can be explained by considering a time-dependent solution $u_{\epsilon}(x, t)$ near the Riemann-Dafermos solution $u_{\epsilon}(x)$. Suppose that $u_{\epsilon}(x, t)$ has $n$ sharp layers near the curves $x_{\epsilon}^{i}(t)$. Using $\xi=\left(x-x_{\epsilon}^{i}(t)\right) / \epsilon$ near the $i$ th singular layer, we have

$$
\epsilon u_{t}=u_{\xi \xi}-\left(D f(u)-x_{\epsilon}^{i}(t)-\frac{d}{d t} x_{\epsilon}^{i}(t)-\epsilon \xi\right) u_{\xi} .
$$

In the initial time layer $0 \leq t \leq \epsilon, u_{t}=O(1 / \epsilon)$ near $x_{\epsilon}^{i}(t)$. We therefore expect to have fast eigenvalues $\lambda=O(1 / \epsilon)$, with the support of the corresponding eigenfunctions near $x_{\epsilon}^{i}(t)$. After the initial time layer, $u_{t}=O(1)$. The solution (if stable) will look like traveling waves in singular layers, and convection in regular layer, where to lowest order $u_{t}+(D f(u)-x I) u_{x}=0$. We expect to have slow eigenvalues of $O(1)$ corresponding to this slow motion.

Fast eigenvalues can be expressed as $\lambda(\epsilon)=\sum_{j=-1}^{\infty} \epsilon^{j} \lambda_{j}$ with $\lambda_{-1} \neq 0$, while slow eigenvalues constitute the special case $\lambda_{-1}=0$. In the $i$ th singular layer, to lowest order, an eigenvalue and corresponding eigenfunction satisfy

$$
\lambda_{-1} U+\left(\left(D f(u(\xi))-x_{0}^{i} I\right) U\right)_{\xi}=U_{\xi \xi} .
$$

If $\lambda_{-1}$ is in the right half of the complex plane, the limiting systems in $\left(U, U_{\xi}\right)$-space at $\xi= \pm \infty$ have exponential dichotomies. If $\lambda_{-1}=0$, however, the limiting systems do not have exponential dichotomies. Instead there is an $n$-dimensional center space, which makes the study of slow eigenvalues more difficult. For an introduction to exponential dichotomies, see [5, 23, 26, 27]. A variant of exponential dichotomies with exponential rate approaching infinity is used in Lemma 5.2.

In [19] conditions for expanding fast eigenvalues and eigenfunctions to any order in $\epsilon$ were given. For slow eigenvalues, however, only the lowest-order terms of the expansions were obtained. In this paper we will show how to successively construct higher-order expansions of slow eigenvalues and eigenfunctions to any desired degree, and we will prove the existence of exact eigenvalues and eigenfunctions near the asymptotic expansions. For an alternate approach to existence of slow eigenvalues and eigenfunctions via geometric singular perturbation theory, see [30]. The latter approach does not yield information about the asymptotic expansions, but does provide geometric insight into the eigenvalue problem.

The assumptions used in [19] will be recalled in $\S 2$. For slow eigenvalues, these assumptions are not sufficient to obtain higher-order expansions. To construct higherorder expansions, we assume that to the lowest-order, the eigenvalue is simple. This is equivalent to assuming that the SLEP function (Evans function) has a simple zero. See $\S 4$ for details. The same condition will enable us in $\S 5$ to construct true 
slow eigenvalue-eigenfunction pairs corresponding to the asymptotic expansions. This condition is also used in [30].

The stability of Riemann-Dafermos solutions is closely related to the stability of Riemann solutions of conservation laws [18] and to the stability of nearby solutions of the usual viscous regularization. At this time our understanding of these relations is incomplete. In [19], it is explained that the fast eigenvalues correspond to eigenvalues of individual viscous shock solutions (traveling waves) of the usual viscous regularization, which have been studied in $[1,39,38,32]$ and elsewhere. The slow eigenvalues are related to inviscid stability of multiple-shock-wave Riemann solutions of hyperbolic conservation laws, which have been studied in $[2,3,14,31]$ and elsewhere. For a system of two equations, formulas for slow eigenvalues of Riemann-Dafermos solutions near a Riemann solution consisting of two Lax shock waves were obtained in [19]. In this case the condition that all slow eigenvalues have negative real part is precisely the same as the condition for BV inviscid stability of the Riemann solution. For a system of more than two equations, the relationship is more complicated, and has recently been elucidated by Lewicka [15].

In a suitable coordinate system, the Dafermos regularization can be viewed as an asymptotic approximation to the usual regularization

$$
u_{T}+f(u)_{X}=u_{X X}
$$

for large $T$. Using the change of variables $x=X / T, t=\ln T$ in (1.9), we obtain

$$
u_{t}+(D f(u)-x I) u_{x}=e^{-t} u_{x x} .
$$

For large $t, e^{-t}$ is small. If we freeze $t=t_{0}$ and let $\epsilon=e^{-t_{0}}$, then we have (1.4), which is a good approximation in a time interval where $e^{-t}$ is close to $e^{-t_{0}}$. Hence the study of the stability of Riemann-Dafermos solutions may provide information about the asymptotic behavior of solutions of (1.10).

The remainder of the paper is organized as follows.

In $\S 2$, we state the assumptions of this paper and recall results from [19].

In $\S 3$, we show that the slow eigenvalue problem can be reduced to a system of equations on regular layers, coupled by jump conditions derived from the singular layers between adjacent regular layers. This approach is similar to the SLEP method (singular limit eigenvalue problem) introduced by Nishiura and Fujii [25] for reactiondiffusion equations. The expansion of the eigenvalue problem to order $\epsilon$ was obtained in [19]. However, the nature of the problem is more fully revealed at order $\epsilon^{2}$. We derive expansions of the eigenvalue problem to all orders in $\epsilon$.

In $\S 4$, the SLEP system is converted to a system of abstract eigenvalue problems. Under the assumption that $-\left(\lambda_{0}+1\right)$ is a simple eigenvalue the abstract system, where $\lambda_{0}$ is the lowest-degree term in the expansion of the slow eigenvalue, we show that formal asymptotic expansions of eigenvalues and eigenfunctions of any order can be constructed recursively. For this purpose we show that the abstract operator is Fredholm, and we characterize its kernel, range, and co-kernel. The SLEP matrix and SLEP function are defined in this section. Our simplicity assumption on $-\left(\lambda_{0}+1\right)$ is equivalent to assuming that $\lambda_{0}$ is a simple zero of the SLEP function. 
A formal asymptotic expansion of eigenvalue (eigenfunction) satisfies the expansion of eigenvalue equations. It becomes a true asymptotic expansion if there exists an exact eigenvalue nearby. Notice that the exact eigenvalue can be constructed using only the zeroth order singular eigenvalue. We discuss asymptotic expansions to any order in this paper for their value in numerical approximation and for the completeness in analysis.

In $\S 5$, under the same assumptions used in $\S 4$, we show that the formal asymptotic expansions obtained in $\S 4$ are true asymptotic expansions of eigenvalue and eigenfunction. The proof is based on the idea of the shadowing lemma of dynamical systems, plus reduction to a SLEP system similar to the one studied in $\S 3$ and $\S 4$. An asymptotic approximation of the eigenfunction to some finite order can be viewed as a pseudo-orbit with small residual and jump errors. Correction terms can be constructed that cancel residual and jump errors to yield an exact eigenvalueeigenfunction pair. Because the linear variational equation about the approximation looks like the recursive equation for computing higher-order expansions, key lemmas obtained in $\S 3$ and $\S 4$ apply in this section also. Since the linear variational system around the pseudo-orbit does not have an exponential dichotomy, one cannot use the shadowing lemma from [4] directly. The part of the solution to which the shadowing lemma does not apply is projected to a center space. The reduced system turns out to be closely related to the SLEP system of $\S 4$.

An important by-product of the analytic approach is that properties of linear operators studied in this paper will be useful in solving (1.4) for initial data near the Riemann-Dafermos solution. After a Laplace transform, the linearized system in the dual variable $s$ is closely related to the eigenvalue problem studied in this paper. When $s$ is not an eigenvalue, the linear operator obtained from Laplace transform is invertible. With some estimates on the transformed solution, the time dependent solution and its stability can be obtained.

I am grateful to the referee for pointing out the work of Suzuki, Nishiura, and Ikeda on a relation between the Evans function and the SLEP method [33].

\section{Assumptions and previous Results}

We first define a so-called structurally stable Riemann solution of (1.2) that consists of exactly $n$ Lax shock waves with speeds $\bar{s}^{1}<\bar{s}^{2}<\cdots<\bar{s}^{n}$. In the new variables $(x, t)=(X / T, \ln T)$, this is a piecewise constant function $u_{0}(x)$ having jumps at $x=\bar{s}^{i}, i=1, \ldots, n$.

A Lax $i$-shock for (1.2) that satisfies the viscous profile criterion is a function

$$
u(x)= \begin{cases}u^{-} & \text {for } x<s, \\ u^{+} & \text {for } x>s,\end{cases}
$$

where $x=\frac{X}{T}$, together with a solution $q(\xi)$ of the traveling wave ODE

$$
\dot{u}=f(u)-f\left(u^{-}\right)-s\left(u-u^{-}\right)
$$

such that:

(L1) $f\left(u^{+}\right)-f\left(u^{-}\right)-s\left(u^{+}-u^{-}\right)=0$. 
(L2) The eigenvalues $\nu_{1}^{-}<\ldots<\nu_{n}^{-}$of $D f\left(u^{-}\right)$satisfy $\nu_{i-1}^{-}<s<\nu_{i}^{-}$.

(L3) The eigenvalues $\nu_{1}^{+}<\ldots<\nu_{n}^{+}$of $D f\left(u^{+}\right)$satisfy $\nu_{i}^{+}<s<\nu_{i+1}^{+}$.

(L4) $q(\xi)$ approaches $u^{-}$as $\xi \rightarrow-\infty$ and $u^{+}$as $\xi \rightarrow \infty$.

Notice that (L1), (L2), and (L3) imply that for (2.2), $u^{ \pm}$are hyperbolic equilibria, the unstable manifold of $u^{-}$has dimension $n-i+1$, and the stable manifold of $u^{+}$ has dimension $i$. Assumption (L4) says that these manifolds intersect. Generically the intersection is a curve (see (S2) or ( $\left.\mathrm{S} 2^{\prime}\right)$ below).

A solution of the Riemann problem (1.2), (1.6), that consists of $n$ Lax shock waves, each satisfying the viscous profile criterion is a piecewise constant function

$$
u_{0}(x)=\bar{u}^{i}, \quad \text { for } \bar{s}^{i}<x<\bar{s}^{i+1}, i=0, \ldots, n,
$$

where $x=\frac{X}{T}$, together with $\mathbb{R}^{n}$-valued functions $q^{i}(\xi), i=1, \ldots, n$, such that:

(R1) $\bar{u}^{0}=u^{\ell}$ and $\bar{u}^{n}=u^{r}$.

(R2) For each $i=1, \ldots, n$, the triple $\left(\bar{u}_{i-1}, \bar{s}_{i}, \bar{u}_{i}\right)$, together with the function $q^{i}(\xi)$, defines a Lax $i$-shock.

Define a mapping $G: \mathbb{R}^{n^{2}+2 n} \rightarrow \mathbb{R}^{n^{2}}$ by

$$
\begin{aligned}
& G\left(u^{0}, s^{1}, u^{1}, \ldots, u^{n-1}, s^{n}, u^{n}\right)= \\
& \left(f\left(u^{1}\right)-f\left(u^{0}\right)-s^{1}\left(u^{1}-u^{0}\right), \ldots, f\left(u^{n}\right)-f\left(u^{n-1}\right)-s^{n}\left(u^{n}-u^{n-1}\right)\right) .
\end{aligned}
$$

Notice that

$$
G\left(\bar{u}^{0}, \bar{s}^{1}, \bar{u}^{1}, \ldots, \bar{u}^{n-1}, \bar{s}^{n}, \bar{u}^{n}\right)=0 .
$$

The Riemann solution just defined is structurally stable provided

(S1) $D G\left(\bar{u}^{0}, \bar{s}^{1}, \bar{u}^{1}, \ldots, \bar{u}^{n-1}, \bar{s}^{n}, \bar{u}^{n}\right)$, restricted to the $n^{2}$-dimensional space of vectors $\left(U^{0}, S^{1}, U^{1}, \ldots, U^{n-1}, S^{n}, U^{n}\right)$ with $U^{0}=U^{n}=0$, is invertible.

(S2) For each $i=1, \ldots, n$, the unstable manifold of $\bar{u}^{i-1}$ and the stable manifold of $\bar{u}^{i}$ for the traveling wave ODE $\dot{u}=f(u)-f\left(\bar{u}^{i-1}\right)-\bar{s}^{i}\left(u-\bar{u}^{i-1}\right)$ meet transversally along $q^{i}(\xi)$.

If $(\mathrm{S} 1)$ and $(\mathrm{S} 2)$ are satisfied, then for each set of Riemann data $\left(u^{0}, u^{n}\right)$ near $\left(\bar{u}^{0}, \bar{u}^{n}\right)$, there is a Riemann solution near the original one.

Condition (S1) can be restated as follows:

$\left(\mathrm{S}^{\prime}\right)$ If we set $\left(U^{0}, U^{n}\right)=(0,0)$, then system of linear equations

$$
\left(D f\left(\bar{u}^{i}\right)-\bar{s}^{i} I\right) U^{i}-\left(D f\left(\bar{u}^{i-1}\right)-\bar{s}^{i} I\right) U^{i-1}-S^{i}\left(\bar{u}^{i}-\bar{u}^{i-1}\right)=0, \quad i=1, \ldots, n,
$$

has only the trivial solution

$$
\left(S^{1}, U^{1}, \ldots, U^{n-1}, S^{n}\right)=(0,0, \ldots, 0,0) .
$$

A condition equivalent to (S2) is the following:

$\left(\mathrm{S} 2^{\prime}\right)$ For each $i=1, \ldots, n$, the linear differential equation

$$
\left(\left(D f\left(q^{i}(\xi)\right)-\bar{s}^{i} I\right) U\right)_{\xi}=U_{\xi \xi}
$$

has, up to scalar multiplication, a unique solution that approaches zero exponentially as $\xi \rightarrow \pm \infty$. It is $q_{\xi}^{i}(\xi)$. 
Asymptotic expansions of Riemann-Dafermos solutions have been obtained under these conditions. Let the location of the $i$ th layer be $x^{i}(\epsilon)$, let the solution in the regular layer (that is, not near $\left.x^{i}(\epsilon)\right)$ be $u_{\epsilon}^{R}(x)$, and let the solution in the $i$ th singular layer (that is, near $\left.x^{i}(\epsilon)\right)$ be $u_{\epsilon}^{i}(\xi)$, where $\xi=\left(x-x^{i}(\epsilon)\right) / \epsilon$. Then

$$
\begin{aligned}
x^{i}(\epsilon) & =\sum \epsilon^{j} x_{j}^{i}, \quad i=1, \ldots, n, \\
u_{\epsilon}^{R}(x) & =\sum \epsilon^{j} u_{j}^{R}(x), \\
u_{\epsilon}^{i}(\xi) & =\sum \epsilon^{j} u_{j}^{i}(\xi) .
\end{aligned}
$$

At lowest order, $x_{0}^{i}=\bar{s}^{i}, u_{0}^{i}(\xi)=q^{i}(\xi)$ and $u_{0}^{R}(x)=\bar{u}^{i}$ is just the piecewise constant Riemann solution of the conservation law (1.5). Furthermore, to all orders of $\epsilon, u_{j}^{R}(x)$ is constant on each regular sublayer $\left(x_{0}^{i}, x_{0}^{i+1}\right), 0 \leq i \leq n$, where $x_{0}^{0}=-\infty, x_{0}^{n+1}=\infty$.

As mentioned in the Introduction, asymptotic expansions of slow eigenvalues and eigenfunctions to lowest order in $\epsilon$ were obtained in [19] and will be outlined below.

\section{Reduction of the eigenvalue Problem to a SLEP System}

In this section, we derive formal expansions of the eigenvalue equations. We also show that at each order $\epsilon^{j}$, the eigenvalue problem can be reduced to a SLEP system.

Define the matrices in regular and singular layers respectively,

$$
\begin{aligned}
D f^{i} & :=D f\left(\bar{u}_{0}^{i}\right), \quad x \in\left[x_{0}^{i}, x_{0}^{i+1}\right], i=0, \ldots n, \\
D f^{i}(\xi) & :=D f\left(q^{i}(\xi)\right), \quad \xi \in \mathbb{R}, i=1, \ldots, n .
\end{aligned}
$$

In the regular layer we will use $D f$ for $D f\left(u_{0}^{R}(x)\right)$ if no confusion should arise.

3.1. Some lemmas. We need to study nonhomogeneous equations as in Lemma 3.1 and Lemma 3.2. Proofs of the two lemmas are deferred to the end of the section. Let $E_{k}$ be the Banach space of continuous functions defined on $\mathbb{R}$ or $\mathbb{R}^{ \pm}$that are $O\left(1+|\xi|^{k}\right)$. Let $E_{\alpha, k}$ be the Banach space of continuous functions that are $O\left(e^{-\alpha|\xi|}\left(1+|\xi|^{k}\right)\right.$ Let the norms of $F \in E_{k}$ and $E_{\alpha, k}$ be

$$
\begin{gathered}
\|F\|_{k}=\sup _{\xi}\left(1+|\xi|^{k}\right)^{-1}|F(\xi)| \\
\|F\|_{\alpha, k}=\sup _{\xi} e^{\alpha|\xi|}\left(1+|\xi|^{k}\right)^{-1}|F(\xi)| .
\end{gathered}
$$

We assume that

$$
0<\alpha<\min \left\{\left|\sigma D f\left(\bar{u}_{0}^{i-1}\right)-x_{0}^{i}\right|,\left|\sigma D f\left(\bar{u}_{0}^{i}\right)-x_{0}^{i}\right|: i=1, \ldots, n\right\} .
$$

Lemma 3.1. Consider the equation

$$
U_{\xi}=\left(D f^{i}(\xi)-x_{0}^{i} I\right) U+G(\xi), \quad \xi \in \mathbb{R} .
$$

If $G_{k} \in E_{k}$, then there exists a unique solution $U_{k} \in E_{k}$ with $U(0) \perp q_{\xi}^{i}(0)$. Denote the solution by $\hat{U}^{i}(\xi, G)$. Moreover,

(1) If there exists $\alpha>0$ such that $G \in E_{\alpha, k}$ for $\xi>0$ or $\xi<0$, then $\hat{U}^{i}(\cdot, G) \in E_{\alpha, k}$ for $\xi>0$ or $\xi<0$.

(2) If $G$ is a polynomial of order $k$, then as $\xi \rightarrow \pm \infty$ respectively, $\hat{U}^{i}(\xi, G)$ approaches 
two polynomials of order $k$.

(3) If $G(\xi)$ approaches two polynomial of order $k$ as $\xi \rightarrow \pm \infty$ respectively, then $\hat{U}^{i}(\xi, G)$ approaches two polynomials of order $k$ as $\xi \rightarrow \pm \infty$. In particular, if $\beta$ is a constant vector, then as $\xi \rightarrow \pm \infty, \hat{U}^{i}(\xi, \beta)$ approaches exponentially the limits

$$
\begin{aligned}
& \hat{U}^{i}(+\infty, \beta)=-\left(D f^{i}-x_{0}^{i} I\right)^{-1} \beta, \\
& \hat{U}^{i}(-\infty, \beta)=-\left(D f^{i-1}-x_{0}^{i} I\right)^{-1} \beta .
\end{aligned}
$$

Lemma 3.2. Consider

$$
U_{\xi \xi}=\left(\left(D f^{i}(\xi)-x_{0}^{i} I\right) U\right)_{\xi}+g(\xi), \quad \xi \in \mathbb{R} .
$$

Assume that there exists a positive integer $k$ such that $g \in E_{k}$. Then

$$
U=\hat{U}^{i}\left(\cdot, \int_{0}^{\xi} g(s) d s\right)
$$

is a particular solution for (3.3) in $E_{k+1}$ with $\|U\|_{k+1} \leq C\|g\|_{k}$. The general solution of (3.3) is

$$
U=\hat{U}^{i}\left(\cdot, \int_{0}^{\xi} g(s) d s\right)+\hat{U}^{i}(\cdot, \beta)+c q_{\xi}^{i},
$$

where the parameters $\beta \in \mathbb{R}^{n}, c \in \mathbb{R}$.

3.2. Formulation of slow eigenvalue problems. Recall that to lowest order, for $i=1, \ldots, n, x_{0}^{i}$ is the location of the $i$ th singular layer, denoted by $S^{i}$. With $x_{0}^{0}=$ $-\infty, x_{0}^{n+1}=\infty$, let $R^{i}=\left(x_{0}^{i}, x_{0}^{i+1}\right)$ be the $i$ th regular sublayer. Figure 3.1 shows the ordering of regular and singular layers.

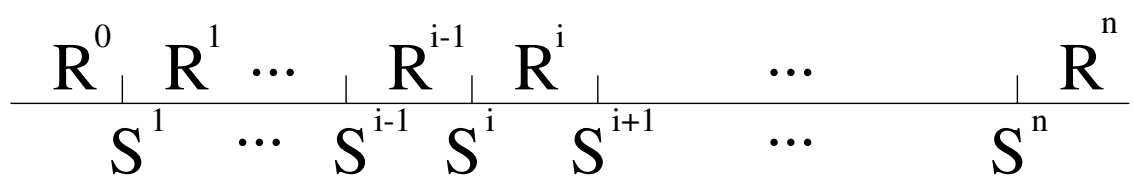

FiguRE 3.1. Ordering of regular and singular layers.

We look for slow eigenvalues and corresponding eigenfunctions of the form

$$
\lambda(\epsilon)=\sum_{j=0}^{\infty} \epsilon^{j} \lambda_{j}, \quad U^{R}(x, \epsilon)=\sum_{j=0}^{\infty} \epsilon^{j} U_{j}^{R}(x), \quad U^{i}(\xi, \epsilon)=\sum_{j=0}^{\infty} \epsilon^{j} U_{j}^{i}(\xi) .
$$

We use $U^{R}$ and $U^{i}$ to denote the function $U$ in regular and singular layers respectively. The stretched variable $\xi=\left(x-x^{i}(\epsilon)\right) / \epsilon$ is used in the $i$ th singular layer. Denote $U^{R}(x, \epsilon)$ and $U_{j}^{R}(x)$ on $R^{i}$ by $U^{R i}(x, \epsilon)$ and $U_{j}^{R i}(x)$ respectively.

Let $C^{k}(\gamma), \gamma>0$ be the space of continuous functions on $\mathbb{R}$ with continuous derivatives up to order $k$, for which the norm

$$
\|U\|_{C^{k}(\gamma)}=\sup _{x}\left\{\left(|U(x)|+\left|U^{\prime}(x)\right|+\cdots+\left|\partial_{x}^{k} U(x)\right|\right) e^{\gamma|x|}\right\} .
$$

is finite. Functions in $C^{k}(\gamma), \gamma>0$ satisfy the decay property

$$
\left|\partial_{j} U(x)\right| \leq C e^{-\gamma|x|}, j \leq k, \text { in the sublayers } R^{0}=\left(-\infty, x_{0}^{1}\right) \text { and } R^{n}=\left(x_{0}^{n}, \infty\right),
$$


for some constant $C$. In [19], it is shown that the initial value problem is well-posed for initial data close to $u(x, \epsilon)$ in $C^{2}(\gamma)$. We also have the following result from [19].

Lemma 3.3. (a) To all orders in $\epsilon$, eigenfunctions $U^{R}(x, \epsilon)$ that satisfy (3.5) are zero in the regular sublayers $R^{0}=\left(-\infty, x_{0}^{1}\right)$ and $R^{n}=\left(x_{0}^{n}, \infty\right)$. That $i s, U_{j}^{R i}=0$ for all $j \geq 0$ and $i=0, n$.

(b) To lowest order, $U^{R}=0$ in the regular layer, i.e., $U_{0}^{R i}=0$ for $0 \leq i \leq n$.

It is known that $\lambda=-1$ is an eigenvalue with eigenfunctions representing shifts in layer positions [19]. Therefore in this paper we assume that $\lambda_{0}+1 \neq 0$.

Let $x^{i}(\epsilon)=\sum \epsilon^{j} x_{j}^{i}$ be the position of the $i$ th singular layer and let $\xi=\left(x-x^{i}(\epsilon)\right) / \epsilon$ in $S^{i}$. The linear variational equation of (1.4) around $u_{\epsilon}$ is

$$
U_{t}+\left(\left(D f\left(u_{\epsilon}\right)-x I\right) U\right)_{x}+U=\epsilon U_{x x} .
$$

Hence the expansions of eigenvalues and eigenfunctions must formally satisfy

$$
\begin{aligned}
(\lambda+1) U^{R}+\left(\left(D f\left(u_{\epsilon}\right)-x I\right) U^{R}\right)_{x} & =\epsilon U_{x x}^{R} \quad \text { in the regular layer, } \\
\epsilon(\lambda+1) U^{i}+\left(\left(D f\left(u_{\epsilon}\right)-x^{i}(\epsilon)-\epsilon \xi I\right) U^{i}\right)_{\xi} & =U_{\xi \xi}^{i} \quad \text { in the singular layer } S^{i}
\end{aligned}
$$

The expansions of eigenfunctions in inner and outer layers satisfy the following matching principle. Let the inner expansions of the two outer layers adjacent to $x^{i}(\epsilon)$ be

$$
\begin{aligned}
& \sum_{0}^{\infty} \epsilon^{j} \tilde{U}_{j}^{i,-}(\xi)=\sum_{0}^{\infty} \epsilon^{j} U_{j}^{R, i-1}\left(\epsilon \xi+x_{0}^{i}+\epsilon x_{1}^{i}+\epsilon^{2} x_{2}^{i}+\ldots\right) \\
& \sum_{0}^{\infty} \epsilon^{j} \tilde{U}_{j}^{i,+}(\xi)=\sum_{0}^{\infty} \epsilon^{j} U_{j}^{R, i}\left(\epsilon \xi+x_{0}^{i}+\epsilon x_{1}^{i}+\epsilon^{2} x_{2}^{i}+\ldots\right) .
\end{aligned}
$$

Note that $U_{0}^{i, \pm}=0$ and $U_{j}^{i, \pm}, j \geq 1$, is a polynomial of degree $j-1$.

Matching Principle: There exists $\alpha>0$ such that

$$
\left|U_{0}^{i}(\xi)\right|+\left|U_{0, \xi}^{i}(\xi)\right| \leq C e^{-\alpha|\xi|}
$$

Moreover, for $j \geq 1$, we have

$$
\begin{array}{cl}
\left|U_{j}^{i}(\xi)-\tilde{U}_{j}^{i,-}(\xi)\right| \leq C\left(1+|\xi|^{j-1}\right) e^{-\alpha|\xi|}, \quad \xi \leq 0 \\
\left|U_{j}^{i}(\xi)-\tilde{U}_{j}^{i,+}(\xi)\right| \leq C\left(1+|\xi|^{j-1}\right) e^{-\alpha|\xi|}, \quad \xi \geq 0 ; \\
U_{j, \xi}^{i}(\xi)-\tilde{U}_{j, \xi}^{i,-}(\xi) \mid \leq C\left(1+|\xi|^{j-1}\right) e^{-\alpha|\xi|}, \quad \xi \leq 0 \\
\left|U_{j, \xi}^{i}(\xi)-\tilde{U}_{j, \xi}^{i,+}(\xi)\right| \leq C\left(1+|\xi|^{j-1}\right) e^{-\alpha|\xi|}, \quad \xi \geq 0 .
\end{array}
$$

If we assume that $\left|U_{j}^{i}(\xi)-\tilde{U}_{j}^{i, \pm}(\xi)\right| \rightarrow 0$ as $\xi \rightarrow \pm \infty$, then the exponential decay rate in (3.9) can be proved by induction. We give the rates explicitly for convenience.

We now prove that (3.10) is a consequence of (3.9).

Lemma 3.4. If the matching conditions (3.9) are satisfied, then the matching of $U_{j, \xi}^{i}(\xi)$ with $\tilde{U}_{j, \xi}^{i, \pm}(\xi)$ for each $j$, as in (3.10), are satisfied also. 
Proof. $\left(\tilde{U}^{i,+}(\xi, \epsilon), \tilde{V}^{i,+}(\xi, \epsilon)\right)$ is a solution of the system

$$
\tilde{U}_{\xi}=\tilde{V}, \quad \tilde{V}_{\xi}=\left(\left(D f\left(u_{\epsilon}\right)-x^{i}(\epsilon)-\epsilon \xi\right) \tilde{U}\right)_{\xi}+\epsilon(\lambda+1) \tilde{U} .
$$

$\left(U^{i}(\xi, \epsilon), V^{i}(\xi, \epsilon)\right)$ is a solution of the system

$$
U_{\xi}^{i}=V^{i}, \quad V_{\xi}^{i}=\left(\left(D f\left(u_{\epsilon}\right)-x^{i}(\epsilon)-\epsilon \xi\right) U^{i}\right)_{\xi}+\epsilon(\lambda+1) U^{i} .
$$

Expanding in powers of $\epsilon$, we find that $\Delta V_{j}:=V_{j}^{i}-\tilde{V}_{j}^{i,+}$ is a solution of

$$
\Delta V_{j, \xi}=\left(D f^{i}(\xi)-x_{0}^{i} I\right) \Delta V_{j}+\Delta h_{j}^{i}(\xi),
$$

where by induction

$$
\left|\Delta h_{j}^{i}(\xi)\right|<C\left(1+|\xi|^{j-1}\right) e^{-\alpha|\xi|}, \quad \xi \geq 0 .
$$

The homogeneous part of the equation has an exponential dichotomy on $\mathbb{R}^{+}$. We also know that $\left|\Delta V_{j}\right| \leq C\left(1+|\xi|^{j-1}\right)$ for $\xi \geq 0$. Therefore, from Lemma 3.1 below,

$$
\left|\Delta V_{j}\right| \leq C\left(1+|\xi|^{j-1}\right) e^{-\alpha|\xi|}, \quad \xi \geq 0 .
$$

A similar proof applies to $\Delta V_{j}:=V_{j}^{i}-\tilde{V}_{j}^{i,-}$

More generally, one can prove the following result.

Lemma 3.5. Under the matching conditions (3.8), (3.9), for any integer $k \geq 0$,

$$
\left|\frac{d^{k}}{d \xi^{k}}\left(U_{j}^{i}-\tilde{U}_{j}^{i, \pm}\right)\right| \leq C\left(1+|\xi|^{j-1}\right) e^{-\alpha|\xi|}, \quad \xi \in \mathbb{R}^{-} \text {or } \mathbb{R}^{+} .
$$

The matching principle (3.9) requires that each $U_{j}^{i}(\xi)$ asymptotically approach a limiting polynomial as $\xi \rightarrow \pm \infty$. For any polynomial $p(\xi)=\sum_{i=0}^{d} c_{i} \xi^{i}, c_{0}$ is called the constant term and the $c_{i} \xi^{i}, i=1, \ldots, d$, are called the non-constant terms.

Definition 3.1. Assume that $\lim _{\xi \rightarrow \pm \infty} U_{j}^{i}(\xi)$ is two polynomials. If the constant terms of the limiting polynomials agree with those of $\tilde{U}_{j}^{i, \pm}$, then we say that the matching is satisfied on constant terms. If coefficients of the non-constant terms agree, then we say the matching is satisfied on non-constant terms.

Using the Taylor expansion

$$
\tilde{U}_{j}^{i,+}(\xi)=U_{j}^{R, i}\left(x_{0}^{i}\right)+D U_{j-1}^{R, i}\left(x_{0}^{i}\right)\left(x_{1}^{i}+\xi\right)+\ldots,
$$

we find that the only contribution of $U_{j}^{R, i}$ to $\tilde{U}_{j}^{i,+}$ is a single term $U_{j}^{R, i}\left(x_{0}^{i}\right)$. Similar expressions hold for $U_{j}^{i,-}(\xi)$. Therefore

$$
\begin{aligned}
& \tilde{U}_{j}^{i,+}(\xi)-U_{j}^{R, i}\left(x_{0}^{i}\right)=\ell \cdot o \cdot t, \\
& \tilde{U}_{j}^{i,-}(\xi)-U_{j}^{R, i-1}\left(x_{0}^{i}\right)=\ell \cdot o \cdot t .
\end{aligned}
$$

Throughout this paper, $\ell \cdot o \cdot t$ (lower order term) denotes terms that involve lower indices and have been obtained in a recursive process (less than $j$ here).

From $U_{0}^{R} \equiv 0$ for all $x$, we find that $\tilde{U}_{0}^{i, \pm}(\xi) \equiv 0$. It can be shown by mathematical induction that $\tilde{U}_{j}^{i, \pm}(\xi)$ is a polynomial of degree $j-1$.

We now expand (3.6) and (3.7) in powers of $\epsilon$. 
At the lowest order $\epsilon^{0}$, we obtain

$$
\begin{array}{lr}
\left(\lambda_{0}+1\right) U_{0}^{R}+\left(\left(D f^{i}-x I\right) U_{0}^{R}\right)_{x}=0 & \text { in the sublayer } R^{i}, \\
\left(\left(D f^{i}(\xi)-x_{0}^{i} I\right) U_{0}^{i}\right)_{\xi}=U_{0 \xi \xi}^{i} & \text { in the singular layer } S^{i} .
\end{array}
$$

Expansion of eigenvalues and eigenfunctions up to order $\epsilon$ has been discussed in [19]. In particular, using the conditions for structural stability, it is shown that at order $\epsilon^{0}$,

$$
U_{0}^{R}(x) \equiv 0, \quad U_{0}^{i}(\xi)=c_{0}^{i} q_{\xi}^{i}(\xi), \quad i=1, \ldots, n .
$$

At order $\epsilon^{1}$, we have

$$
\begin{aligned}
& \left(\lambda_{0}+1\right) U_{1}+\left(\left(D f^{i}-x I\right) U_{1}\right)_{x}=0 \quad \text { in } R^{i}, \\
& \left(\lambda_{0}+1\right) U_{0}^{i}+\left(\left(D^{2} f\left(q^{i}\right) u_{1}^{i}-\left(x_{1}^{i}+\xi\right) I\right) U_{0}^{i}\right)_{\xi} \\
& +\left(\left(D f^{i}(\xi)-x_{0}^{i} I\right) U_{1}^{i}\right)_{\xi}=U_{1 \xi \xi}^{i} \quad \text { in } S^{i} .
\end{aligned}
$$

The matching principle yields

$$
U_{1}^{i}(\xi) \rightarrow \begin{cases}U_{1}^{R}\left(x_{0}^{i}-\right) & \xi \rightarrow-\infty \\ U_{1}^{R}\left(x_{0}^{i}+\right) & \xi \rightarrow \infty\end{cases}
$$

Let $\Delta^{i}=\bar{u}_{0}^{i}-\bar{u}_{0}^{i-1}$. Then $\int_{-\infty}^{\infty} q_{\xi}^{i} d \xi=\Delta^{i}$. Integrating (3.16), we have the jump condition of $U_{1}^{R}$ at $x_{0}^{i}$ :

$$
\begin{aligned}
\left(\lambda_{0}+1\right) c_{0}^{i} \Delta^{i}+\left(D f^{i}-x_{0}^{i} I\right) & U_{1}^{R}\left(x_{0}^{i}+\right) \\
& -\left(D f^{i-1}-x_{0}^{i} I\right) U_{1}^{R}\left(x_{0}^{i}-\right)=0, \quad i=1, \ldots, n .
\end{aligned}
$$

It is shown in [19] that if we can find

$$
\left(\lambda_{0}, c_{0}^{1}, \ldots, c_{0}^{n}, U_{1}^{R}(x)\right)
$$

satisfying (3.15), (3.17) with $U_{1}^{R}(x)=0$ on $R^{0} \cup R^{n}$, then there exist $U_{1}^{i}(\xi), 1 \leq i \leq n$, that satisfy (3.16) and matching condition (3.9).

Although the expansion at order $\epsilon^{2}$ is a special case of $\epsilon^{j}, j \geq 2$, it is presented in detail to help illustrate the idea.

At order $\epsilon^{2}$, we have

$$
\begin{gathered}
\left(\lambda_{0}+1\right) U_{2}^{R}+\lambda_{1} U_{1}^{R}+\left(\left(D f^{i}-x I\right) U_{2}^{R}\right)_{x}+\left(D^{2} f\left(\bar{u}_{0}^{i}\right) \bar{u}_{1}^{i} U_{1}\right)_{x}=U_{1 x x}^{R}, \\
\left(\lambda_{0}+1\right) U_{1}^{i}+\lambda_{1} U_{0}^{i}+\left(\left(D f^{i}(\xi)-x_{0}^{i} I\right) U_{2}^{i}\right)_{\xi}+\left(\left(D^{2} f\left(q^{i}\right) u_{1}^{i}-x_{1}^{i}-\xi\right) U_{1}^{i}\right)_{\xi} \\
+\left(\left(D^{3} f\left(q^{i}\right)\left(u_{1}^{i}\right)^{2} / 2+D^{2} f\left(q^{i}\right) u_{2}^{i}-x_{2}^{i}\right) U_{0}^{i}\right)_{\xi}=U_{2 \xi \xi}^{i} .
\end{gathered}
$$

At order $\epsilon^{j}$, we have

$$
\begin{aligned}
& \left(\lambda_{0}+1\right) U_{j}^{R}+\lambda_{j-1} U_{1}^{R}+\left(\left(D f^{i}-x I\right) U_{j}^{R}\right)_{x}=W_{j}^{i}, \\
& \left(\lambda_{0}+1\right) U_{j-1}^{i}+\lambda_{j-1} U_{0}^{i}+\left(\left(D f^{i}(\xi)-x_{0}^{i} I\right) U_{j}^{i}\right)_{\xi}+H_{j}^{i}(\xi)=U_{j \xi \xi}^{i} .
\end{aligned}
$$

$W_{j}^{i}$ and $H_{j}^{i}$ involve terms with indices lower than $j$ only, and can be expressed as Taylor polynomials of $U_{k}^{R i}, U_{k}^{i}, k \leq j-1$.

We can think $\left(U_{1}^{R}, U_{0}^{i}\right)$ as an eigenfunction for system (3.15), (3.16) corresponding to the eigenvalue $\left(\lambda_{0}+1\right)$. In System (3.20), (3.21), we solve for $\left(U_{j}^{R}, U_{j}^{i}\right)$ with a 
undetermined parameter $\lambda_{j-1}$ that is multiplied to the eigenfunction $\left(U_{1}^{R}, U_{0}^{i}\right)$. If this system defines a Fredholm operator, then to construct higher order expansions, we need conditions that ensure the eigenvalue $\lambda_{0}+1$ is simple (or at least semi-simple). However, we will not show that this linear system is Fredholm. Instead, we will show in the next section that the system can be reduced to a lower dimensional system (SLEP) and that the reduced system is Fredholm. The simpleness of $\lambda_{0}+1$ will be imposed on the reduced lower dimensional system.

3.3. Reduction of the eigenvalue problem to a SLEP system. As is done in [19] at order $\epsilon$, we first want to show that for any order $\epsilon^{j}$, systems (3.20), (3.21) and the matching condition (3.9) can be reduced to a system of equations in regular layers and a set of jump conditions across singular layers. The reduced system is similar to the SLEP system introduced by Nishiura and Fujii [25].

Observe that the non-homogeneous terms in (3.19) are $O(1)$. Naturally, we look for $\left|U_{2}^{i}(\xi)\right|=O(1+|\xi|)$. Using Lemma 3.2 and induction, we can show that $\left|U_{j}^{i}(\xi)\right|=$ $O\left(1+|\xi|^{j-1}\right)$.

As $\xi \rightarrow \pm \infty, U_{2}^{i}(\xi)$ must match with $\tilde{U}_{2}^{i, \pm}(\xi)$, which is a first-order polynomial on each side of $x_{0}^{i} \pm$. In Lemma 3.6 below, we show that the matching of coefficients of the first-degree powers of $\xi$ is automatically satisfied. Only the constants must be matched at each recursive step.

At the expansion of order $\epsilon^{2}$, we assume that $U_{1}^{i}=U_{1}^{i \perp}+c_{1}^{i} q_{\xi}^{i}$, where $U_{1}^{i \perp}$ has been uniquely obtained with $U_{1}^{i \perp}(0) \perp q_{\xi}^{i}(0)$ while $c_{1}^{i}$ is undetermined. By integrating (3.19) and matching the constant terms, we obtain the jump condition of $U_{2}^{R}(x)$ at $x=x_{0}^{i}$ :

$$
\left(\lambda_{0}+1\right) c_{1}^{i} \Delta^{i}+\lambda_{1} c_{0}^{i} \Delta^{i}+\left(D f^{i}-x_{0}^{i} I\right) U_{2}^{R}\left(x_{0}^{i}+\right)-\left(D f^{i-1}-x_{0}^{i} I\right) U_{2}^{R}\left(x_{0}^{i}-\right)=J_{2}^{i} .
$$

Here $J_{2}^{i}$ involves $U_{0}^{i}, U_{1}^{i \perp}$ and $U_{1, x}^{R}\left(x_{0}^{i} \pm\right)$ only and is a special case of (3.23).

As $\xi \rightarrow \pm \infty, U_{j}^{i}(\xi)$ must match with $\tilde{U}_{j}^{i, \pm}(\xi)$, which by induction is a polynomial of order $j-1$ on each side of $x_{0}^{i}$. Meanwhile, since $H_{j}^{i}$ is a Taylor polynomial of $U_{k}^{i}, k \leq$ $j-2$ and $U_{j-1}^{i \perp}$, by induction it can be shown that $H_{j}^{i}(\xi)$ approaches polynomials at $\xi= \pm \infty$ respectively. By Lemma 3.2, $U_{j}^{i}(\xi)$ approaches polynomials as $\xi \rightarrow \pm \infty$. In Lemma 3.6, we will show that the matching of coefficients of the non-constant terms is automatically satisfied. From Lemma 3.2, the solution of (3.21) can be expressed as

$$
U_{j}^{i}=\hat{U}^{i}\left(\cdot, \int_{0}^{\xi}\left(\left(\lambda_{0}+1\right) U_{j-1}^{i}+\lambda_{j-1} U_{0}^{i}+H_{j}^{i}\right) d \xi\right)+\hat{U}^{i}\left(\cdot, \beta_{j}^{i}\right)+c_{j}^{i} q_{\xi}^{i} .
$$

The parameters $\beta_{j}^{i}$ and $c_{j}^{i}$ are undetermined.

By the induction assumption, $U_{j-1}^{i}$ and $H_{j}^{i}(\xi)$ approaches polynomials of degree $j-2$ as $\xi \rightarrow \pm \infty$.

In this paper we often encounter functions that approach polynomials as $\xi \rightarrow$ $\pm \infty$, and we are interested in the constant terms of the limiting polynomials. For convenience, we introduce the following notation. Let $Q_{c}$ be an operator that projects a polynomial to its constant term, i.e.,

$$
Q_{c}\left(c_{0}+c_{1} \xi+\cdots+c_{l} \xi^{k}\right)=c_{0} .
$$


If $g(\xi) \rightarrow P(\xi)=c_{0}+c_{1} \xi+\cdots+c_{l} \xi^{k}$ as $\xi \rightarrow-\infty$ or $\infty$, then define

$$
Q_{c}(g( \pm \infty)):=Q_{c}(P)=c_{0} \text {. }
$$

We now introduce a recursive procedure that reduces the coupled inner-outer system to a system of equations in outer layers $\left\{R^{i}\right\}_{0}^{n+1}$ coupled with a set of jump conditions between two adjacent outer layers $R^{i-1}, R^{i}, i=1, \ldots, n$. The reduced system is called the SLEP system following Fujii and Nishiura. We will derive the equations in $R^{i}$ and the jump conditions. We also will show inductively that if the SLEP system can be solved, then we can find $U_{\ell}^{i}, i=1, \ldots, n$, in the inner layers so that the matching conditions (3.9), (3.10) are satisfied. To this end we need two lemmas. The first shows that the matching of non-constant terms is always satisfied. The second shows that the matching of constant terms can be achieved by choosing a parameter in solving the inner systems for $U_{\ell}^{i}$. In fact, we derive the jump conditions across singular layers based on the matching of constant terms only. (Otherwise we would have too many jump conditions, each for a particular power of $\xi$.)

Define $J_{j}^{i}$ as follows:

$$
\begin{aligned}
-J_{j}^{i}: & =Q_{c}\left(\int_{0}^{\infty} H_{j}^{i}(\xi) d \xi\right)-Q_{c}\left(\int_{0}^{-\infty} H_{j}^{i}(\xi) d \xi\right) \\
& +\left(\lambda_{0}+1\right)\left(Q_{c}\left(U_{j-1}^{i \perp}(\infty)\right)-Q_{c}\left(U_{j-1}^{i \perp}(-\infty)\right)\right. \\
& +\left(D f^{i}-x_{0}^{i} I\right) Q_{c}\left(\tilde{U}_{j}^{i,+}\left(x_{0}^{i}\right)-U_{j}^{R, i}\left(x_{0}^{i}\right)\right) \\
& -\left(D f^{i-1}-x_{0}^{i} I\right) Q_{c}\left(\tilde{U}_{j}^{i,-}\left(x_{0}^{i}\right)-U_{j}^{R, i-1}\left(x_{0}^{i}\right)\right) \\
& -Q_{c}\left(\tilde{U}_{j, \xi}^{i,+}\left(x_{0}^{i}\right)-\tilde{U}_{j, \xi}^{i,-}\left(x_{0}^{i}\right)\right) .
\end{aligned}
$$

By (3.12), $\tilde{U}_{j}^{i,+}\left(x_{0}^{i}\right)-U_{j}^{R, i}\left(x_{0}^{i}\right)$ and $\tilde{U}_{j}^{i,-}\left(x_{0}^{i}\right)-U_{j}^{R, i-1}\left(x_{0}^{i}\right)$ only involve lower-order terms. Also by differentiating (3.11), we find that $\tilde{U}_{j, \xi}^{i,+}\left(x_{0}^{i}\right)-\tilde{U}_{j, \xi}^{i,-}\left(x_{0}^{i}\right)$ only involves $U_{k, x}^{R}$ for $k \leq j-1$. Thus $J_{j}^{i}$ can be calculated from terms with indices lower than $j$.

The jump condition on $U_{j}^{R}$ at $x_{0}^{i}$ comes from the matching of constant terms:

$$
\begin{aligned}
\left(\lambda_{0}+1\right) c_{j-1}^{i} \Delta^{i}+\lambda_{j-1} c_{0}^{i} \Delta^{i} & \\
& +\left(D f^{i}-x_{0}^{i} I\right) U_{j}^{R}\left(x_{0}^{i}+\right)-\left(D f^{i-1}-x_{0}^{i} I\right) U_{j}^{R}\left(x_{0}^{i}-\right)=J_{j}^{i} .
\end{aligned}
$$

Lemma 3.6. Assume that the expansion of eigenfunctions $\left(U_{j}^{R}, U_{j}^{i \perp}, c_{j}^{i}, \lambda_{j}\right), j \leq \ell-2$, and $\left(U_{\ell-1}^{R}, U_{\ell-1}^{i \perp}\right)$ have been computed such that they satisfy systems (3.20), (3.21) and the matching principle (3.9), (3.10) up to $j \leq \ell-1$. If $\left(U_{\ell}^{R}, \lambda_{\ell-1}, c_{\ell-1}^{i}\right)$ have been obtained that satisfy (3.20), (3.24) with any $J_{j}^{i}$, and $U_{\ell}^{i}$ is a solution of (3.21) as in (3.22) with any $\beta_{\ell}^{i}$ and $c_{\ell}^{i}$, then the matching as in (3.9), (3.10) with $j=\ell$ is always satisfied for non-constant terms. Moreover,

$$
\frac{d}{d \xi}\left(U_{\ell}^{i}(\xi)-\tilde{U}_{\ell}^{i, \pm}(\xi)\right) \rightarrow 0, \quad \xi \rightarrow \pm \infty
$$

Proof. Except for the constant terms, we show that other higher-order terms in $U_{\ell}^{i}(\xi)-\tilde{U}_{\ell}^{i, \pm}(\xi)$ are always matched, regardless the choice of $\beta_{\ell}^{i}$. The proof here is similar to $[9,16]$. 
Consider the formal expansion of $\tilde{U}(\xi, \epsilon)$, the inner expansion of outer solutions. We find that $\tilde{U}_{\ell}^{i,+}$ satisfies an equation similar to that of $U_{\ell}^{i}$ :

$$
\left.\left(\lambda_{0}+1\right) \tilde{U}_{\ell-1}^{i}+\lambda_{\ell-1} \tilde{U}_{0}^{i}+\left(D f^{i}-x_{0}^{i} I\right) \tilde{U}_{\ell}^{i}\right)_{\xi}+\tilde{H}_{\ell}^{i}(\xi)=\tilde{U}_{\ell \xi \xi}^{i},
$$

where $\tilde{H}_{\ell}^{i}$ can be expressed as a Taylor polynomial of $\tilde{U}_{j}^{i}, j \leq \ell-1$. Comparing the above with (3.21), we find that the difference $\Delta U:=U_{\ell}^{i}-\tilde{U}_{\ell}^{i}$ satisfies

$$
\begin{aligned}
& \Delta U_{\xi \xi}=\left(\left(D f^{i}(\xi)-x_{0}^{i} I\right) \Delta U\right)_{\xi}+\left(\lambda_{0}+1\right)\left(U_{\ell-1}^{i}-\tilde{U}_{\ell-1}^{i}\right) \\
& \quad+\lambda_{\ell-1}\left(U_{1}^{i}-\tilde{U}_{1}^{i}\right)+H_{\ell}^{i}(\xi)-\tilde{H}_{\ell}^{i}(\xi)+\left(\left(D f^{i}(\xi)-D f^{i}\right) \tilde{U}_{\ell}^{i}(\xi)\right)_{\xi}
\end{aligned}
$$

By the induction assumption, the forcing term $H_{\ell}^{i}(\xi)$ approaches $\tilde{H}_{\ell}^{i}(\xi)$ exponentially as $\xi \rightarrow \infty$. Also observe that $D f^{i}(\xi) \rightarrow D f^{i}$ as $\xi \rightarrow \infty$. From Lemmas 3.2 and 3.1 ,

$$
\Delta U=U_{\ell}^{i}(\xi)-\tilde{U}_{\ell}^{i,+}(\xi) \rightarrow \text { constant as } \xi \rightarrow \infty
$$

Similarly,

$$
U_{\ell}^{i}(\xi)-\tilde{U}_{\ell}^{i,-}(\xi) \rightarrow \text { constant as } \xi \rightarrow-\infty .
$$

Therefore the matching of non-constant terms is satisfied for any $\beta_{\ell}^{i}$.

Integrating (3.25), we find that $\Delta U_{\xi}(\xi)$ approaches constants as $|\xi| \rightarrow \infty$. The constants must be zero, or $\Delta U(\xi)$ would not have constant limits as $|\xi| \rightarrow \infty$.

Lemma 3.7. Under the same hypotheses as Lemma 3.6, if $\left(U_{\ell}^{R}, \lambda_{\ell-1}, c_{\ell-1}^{i}\right)$ is a solution to the reduced system (3.20), (3.24) for $j=\ell$, with $J_{\ell}^{i}$ as in (3.23), then we can choose $\beta_{\ell}^{i}$ so that the solution $U_{\ell}^{i}$ of $(3.21)$ as in (3.22) satisfies the matching principle (3.9), (3.10) with $j=\ell$.

Proof. Assume that we have constructed $\left(U_{j}^{R}(x), U_{j}^{i}(\xi)\right), j \leq \ell-1$, and $\lambda_{j}, j \leq \ell-2$, that satisfy the formal equations and matching conditions, except for the term $U_{\ell-1}^{i}$, which has the form

$$
U_{\ell-1}^{i}=U_{\ell-1}^{i \perp}+c_{\ell-1}^{i} q_{\xi}^{i},
$$

where $U_{\ell-1}^{i \perp}$ satisfies $U_{\ell-1}^{i \perp}(0) \perp q_{\xi}^{i}(0)$ and is determined but $c_{\ell-1}^{i}$ is still undetermined. We look for $\left(U_{\ell}^{R}, U_{\ell}^{i}, \lambda_{\ell-1}\right)$ that satisfies (3.20), (3.21) and the matching condition (3.9) at $j=\ell$. At the same time we also determine $c_{\ell-1}^{i}$.

In the next section, we solve for $U_{\ell}^{R}(x), \lambda_{\ell-1}$ from (3.20) with $j=\ell$ and the jump condition (3.24) with $J_{\ell}^{i}$ defined in (3.23). Here we assume that this is done and we construct the inner expansion $U_{\ell}^{i}$ that satisfies (3.21) and the matching condition (3.9) at $j=\ell$.

Substituting $J_{\ell}^{i}$ from (3.23) into (3.24) with $j=\ell$, and using

$$
Q_{c}\left(U_{\ell-1}^{i}(\infty)\right)-Q_{c}\left(U_{\ell-1}^{i}(-\infty)\right)=Q_{c}\left(U_{\ell-1}^{i \perp}(\infty)\right)-Q_{c}\left(U_{\ell-1}^{i \perp}(-\infty)\right)+c_{\ell-1}^{i} \Delta^{i}
$$


we have

$$
\begin{aligned}
& Q_{c}\left(\int_{0}^{\infty} H_{\ell}^{i}(\xi) d \xi\right)-Q_{c}\left(\int_{0}^{-\infty} H_{\ell}^{i}(\xi) d \xi\right) \\
& +\left(\lambda_{0}+1\right)\left(Q_{c}\left(U_{\ell-1}^{i}(\infty)\right)-Q_{c}\left(U_{\ell-1}^{i}(-\infty)\right)\right. \\
& +\lambda_{\ell-1}\left(U_{0}^{i}(\infty)-U_{0}^{i}(-\infty)\right) \\
& +\left(D f^{i}-x_{0}^{i} I\right) Q_{c}\left(\tilde{U}_{\ell}^{i,+}\left(x_{0}^{i}\right)\right) \\
& -\left(D f^{i-1}-x_{0}^{i} I\right) Q_{c}\left(\tilde{U}_{\ell}^{i,-}\left(x_{0}^{i}\right)\right) \\
& =Q_{c}\left(\tilde{U}_{\ell, \xi}^{i,+}\left(x_{0}^{i}\right)-\tilde{U}_{\ell, \xi}^{i,-}\left(x_{0}^{i}\right)\right) .
\end{aligned}
$$

Now solve (3.21) for $U_{\ell}^{i}$. Using Lemma 3.2, the solution can be expressed as (3.22) with $j=\ell$. The limits of $c_{\ell}^{i} q_{\xi}^{i}(\xi), \xi \rightarrow \pm \infty$, are both zero and do not affect the matching. Therefore the value of $c_{\ell}^{i}$ cannot be determined from the expansion to order $\epsilon^{\ell}$.

Plug $U_{\ell}^{i}$ as in (3.22) into (3.21) and apply the integral operator $\int_{0}^{\xi} \cdot d \xi$ to (3.21). Observing that all the terms approach polynomials as $\xi \rightarrow \pm \infty$, and keeping only the constants in the limit, we find the jump of constants between the two limits is:

$$
\begin{aligned}
& Q_{c}\left(\int_{0}^{\infty} H_{\ell}^{i}(\xi) d \xi\right)-Q_{c}\left(\int_{0}^{-\infty} H_{\ell}^{i}(\xi) d \xi\right) \\
& +\left(\lambda_{0}+1\right)\left(Q_{c}\left(U_{\ell-1}^{i}(\infty)\right)-Q_{c}\left(U_{\ell-1}^{i}(-\infty)\right)\right. \\
& +\lambda_{\ell-1}\left(U_{0}^{i}(\infty)-U_{0}^{i}(-\infty)\right) \\
& +\left(D f^{i}-x_{0}^{i} I\right) Q_{c}\left(U_{\ell}^{i}(\infty)\right) \\
& -\left(D f^{i-1}-x_{0}^{i} I\right) Q_{c}\left(U_{\ell}^{i}(-\infty)\right) \\
& =Q_{c}\left(U_{\ell, \xi}^{i}(\infty)-U_{\ell, \xi}^{i}(-\infty)\right) .
\end{aligned}
$$

Recall that from the last statement of Lemma 3.6,

$$
Q_{c} U_{\ell, \xi}^{i}(\infty)=Q_{c} \tilde{U}_{\ell, \xi}^{i,+}\left(x_{0}^{i}\right), \quad Q_{c} U_{\ell, \xi}^{i}(-\infty)=Q_{c} \tilde{U}_{\ell, \xi}^{i,-}\left(x_{0}^{i}\right) .
$$

Comparing (3.26) and (3.27), we have

$$
\left(D f^{i}-x_{0}^{i} I\right)\left(Q_{c}\left(U_{\ell}^{i}(\infty)\right)-Q_{c}\left(\tilde{U}_{\ell}^{i,+}\left(x_{0}^{i}\right)\right)\right)=\left(D f^{i-1}-x_{0}^{i} I\right)\left(Q_{c}\left(U_{\ell}^{i}(-\infty)\right)-Q_{c}\left(\tilde{U}_{\ell}^{i,-}\left(x_{0}^{i}\right)\right)\right) \text {. }
$$

We choose $\beta_{\ell}^{i}$ so that the constant terms of $U_{\ell}^{i}(+\infty)$ and $\tilde{U}_{\ell}^{i,+}(\xi)$ are matched. To this end, observe that from $(3.22)$,

$$
Q_{c}\left(U_{\ell}^{i}(\infty)\right)=Q_{c}\left(\hat{U}^{i}\left(\infty, \int_{0}^{\xi}\left(\left(\lambda_{0}+1\right) U_{\ell-1}^{i}+\left(\lambda_{\ell-1}+1\right) U_{0}^{i}+H_{\ell}^{i}\right) d \xi\right)\right)+\hat{U}^{i}\left(\infty, \beta_{\ell}^{i}\right) .
$$

The first term above is independent of $\beta_{\ell}^{i}$, but the second term $\hat{U}^{i}\left(\infty, \beta_{\ell}^{i}\right)=-\left(D f^{i}-\right.$ $\left.x_{0}^{i} I\right)^{-1} \beta_{\ell}^{i}$. There is a unique value of $\beta_{\ell}^{i}$ such that $Q_{c}\left(U_{\ell}^{i}(\infty)\right)=Q_{c}\left(\tilde{U}_{\ell}^{R,+}\right)$. Choosing this $\beta_{\ell}^{i}$, we have $Q_{c}\left(U_{\ell}^{i}(\infty)\right)-Q_{c}\left(\tilde{U}_{\ell}^{i,+}\left(x_{0}^{i}\right)\right)=0$. From $(3.28)$, we have $Q_{c}\left(U_{\ell}^{i}(-\infty)\right)-$ $Q_{c}\left(\tilde{U}_{\ell}^{i,-}\left(x_{0}^{i}\right)\right)=0$. The matching of constant terms at both ends has been achieved. 
We summarize the reduction to a SLEP system in Theorem 3.8. Note that the original SLEP method of Fujii and Nishiura dealt with the lowest-order expansion of eigenvalues and eigenfunctions, whereas we give a recursive procedure for expansions to any desired order.

Theorem 3.8. Assume that the expansion of eigenfunctions $\left(U_{j}^{R}, U_{j}^{i \perp}, c_{j}^{i}, \lambda_{j}\right), j \leq$ $\ell-2$, and $\left(U_{\ell-1}^{R}, U_{\ell-1}^{i \perp}\right)$ have been computed such that they satisfy systems (3.20), (3.21) and the matching principle (3.9), (3.10) up to $j \leq \ell-1$. If $\left(U_{\ell}^{R}, \lambda_{\ell-1}, c_{\ell-1}^{i}\right)$ is a solution to the reduced system (3.20), (3.24) at $j=\ell$, with $J_{\ell}^{i}$ as in (3.23), then there exists a unique $\beta_{\ell}^{i}$ so that the solution $U_{\ell}^{i}=U_{\ell}^{i \perp}+c_{\ell}^{i} q_{\xi}^{i}$ of (3.21) as in (3.22) satisfies the matching principle (3.9), (3.10) with $j=\ell$. The parameters $c_{\ell}^{i}, i=1, \ldots, n$ remain undetermined.

Remark 3.1. In [19] we defined $V(x)=\left(D f\left(u_{0}^{R}(x)\right)-x I\right) U_{j}^{R}(x)$. Then $V(x)$ is piecewise $C^{1}$ and has a jump at each layer position $x_{0}^{i}$. The singular layer simply provides a delta function type forcing to the equation in the regular layer (3.20). This was the point of view of Nishiura and Fujii, who introduced the SLEP system [25]. The idea of SLEP is also used in $[9,16]$.

Proof of Lemma 3.1. Since the homogeneous part of the limiting systems of (3.1)

$$
U_{\xi}=\left(D f^{i}-x_{0}^{i} I\right) U, \quad U_{\xi}=\left(D f^{i-1}-x_{0}^{i} I\right) U,
$$

has real nonzero eigenvalues, the homogeneous part of (3.1) has exponential dichotomies on $\mathbb{R}^{ \pm}$respectively $[5,23]$. Let the the principal matrix solution be denoted $S(\xi, \eta)$ and the stable and unstable projections related to the dichotomies be $P_{s}(\xi)+P_{u}(\xi)=I$. Solutions on $\mathbb{R}^{ \pm}$can be expressed as

$$
\begin{aligned}
& U(\xi)=S(\xi, 0) \phi_{s}(0)+\int_{0}^{\xi} S(\xi, \eta) P_{s}(\eta) G(\eta) d \eta+U^{+}(\xi), \quad \xi \geq 0 \\
& U(\xi)=S(\xi, 0) \phi_{u}(0)+\int_{0}^{\xi} S(\xi, \eta) P_{u}(\eta) G(\eta) d \eta+U^{-}(\xi), \quad \xi \leq 0
\end{aligned}
$$

where

$$
\begin{aligned}
U^{+}(\xi) & =\int_{\infty}^{\xi} T^{i}(\xi, \eta) P_{s}(\eta) G(\eta) d \eta, \\
U^{-}(\xi) & =\int_{-\infty}^{\xi} T^{i}(\xi, \eta) P_{u}(\eta) G(\eta) d \eta, \\
\phi_{s}(0) & =P_{s}(0) U(0), \quad \phi_{u}(0)=P_{u}(0) U(0) .
\end{aligned}
$$

The solution $U(\xi), \xi \in \mathbb{R}$ can be found by matching $U^{ \pm}$at $\xi=0$ as follows.

To have $U(0-)=U(0+)$, we need

$$
U^{+}(0)+\phi_{s}(0)=U^{-}(0)+\phi_{u}(0) .
$$

Since that unstable subspace of the dichotomy on $\mathbb{R}^{-}$and the stable subspace of the dichotomy on $\mathbb{R}^{+}$intersect transversely, we can find $\phi_{u}$ and $\phi_{s}$ such that

$$
\phi_{s}(0)-\phi_{u}(0)=U^{-}(0)-U^{+}(0) \text {. }
$$


The choice of $\left(\phi_{u}, \phi_{s}\right)$ is unique if we require that $U^{+}(0)+\phi_{s}(0) \perp q_{\xi}^{i}(0)$.

The proof of (1) is left to the reader.

To prove (2), assume that $G$ is a polynomial of order $k$. Let $\tilde{U}^{+}$be the polynomial solution to the equation

$$
U_{\xi}=\left(D f^{i}-x_{0}^{i} I\right) U+G .
$$

Let $\Delta U=\hat{U}(\cdot, G)-\tilde{U}^{+}$. Then

$$
(\Delta U)_{\xi}=\left(D f^{i}(\xi)-x_{0}^{i} I\right)(\Delta U)+\left(D f^{i}(\xi)-D f^{i}\right) \tilde{U}^{+} .
$$

Since for $\xi \geq 0$ the forcing term for the equation of $\Delta U$ is

$$
\left(D f^{i}(\xi)-D f^{i}\right) \tilde{U}=O\left(e^{-\alpha|\xi|}\left(1+|\xi|^{k}\right)\right.
$$

we have $|\Delta U|=O\left(e^{-\alpha|\xi|}\left(1+|\xi|^{k}\right), \xi \geq 0\right.$.

Similarly, let $\tilde{U}^{-}$be the polynomial solution to

$$
U_{\xi}=\left(D f^{i-1}-x_{0}^{i} I\right) U+G .
$$

We have $\left|\hat{U}^{i}(\xi, G)-\tilde{U}^{-}\right|=O\left(e^{-\alpha|\xi|}\left(1+|\xi|^{k}\right), \xi \leq 0\right.$.

The proof of (3) can be achieved by combining the results of (1) and (2). (3.2) can be derived by observing that $D f^{i}(\xi) \rightarrow D f^{i}$ as $\xi \rightarrow \infty$ and $D f^{i}(\xi) \rightarrow D f^{i-1}$ as $\xi \rightarrow-\infty$ in $(3.1)$.

Proof of Lemma 3.2. Let $G(\xi)=\int_{0}^{\xi} g(s) d s$. Then $G(\xi) \leq C\left(1+|\xi|^{k+1}\right)$. Integrating (3.3), we have

$$
U_{\xi}=\left(D f^{i}(\xi)-x_{0}^{i} I\right) U+G(\xi)+\beta .
$$

The general solution of (3.3) can be obtained from the superposition principle.

\section{EXPANSIONS OF EIGENVALUE AND EIGENFUNCTIONS}

The procedure of recursively computing $U_{j}^{R}, \lambda_{j}$ and $c_{j-1}^{i}$ that satisfy (3.20) and (3.24), with $J_{j}^{i}$ defined by (3.23), is equivalent to a system of eigenvalue problems of abstract operators. In (H1) below, the simpleness of the eigenvalue will be imposed on the abstract problem. Generalization to the case that the eigenvalue is semi-simple is straitforward in the abstract setting and will not be discussed in this paper.

Let $\mathcal{H}$ be the linear space of sequences of $n$-vectors

$$
\mathcal{H}:=\left\{\left\{h^{i}\right\}_{1}^{n} \mid h^{i} \in \mathbb{R}^{n}\right\} .
$$

Let $\mathcal{H}_{1}$ be a subspace of $\mathcal{H}$ defined as:

$$
\mathcal{H}_{1}:=\left\{\left\{c^{i} \Delta^{i}\right\}_{1}^{n} \mid c^{i} \in \mathbb{R}\right\} .
$$

Let $\mathcal{E}$ be the linear space of $n+1$ continuous functions each defined on $R^{i}=$ $\left[x_{0}^{i}, x_{0}^{i+1}\right], i=0, \ldots, n$ :

$$
\mathcal{E}:=\left\{\left\{U^{i}\right\}_{0}^{n} \mid U^{i}(x) \text { is continuous on } R^{i}, U^{0} \equiv 0, U^{n} \equiv 0\right\} .
$$

Let $\mathcal{E}_{1}$ be a subspace of $\mathcal{E}$ defined as

$$
\mathcal{E}_{1}:=\left\{\left\{U^{i}\right\}_{0}^{n} \in \mathcal{E} \mid U^{i} \in C^{1}\left(R^{i}\right)\right\} .
$$


Let $\mathcal{L}:\left(\mathcal{E}_{1}, \mathcal{H}_{1}\right) \rightarrow(\mathcal{E}, \mathcal{H})$ be a linear operator defined as follows:

$$
\begin{aligned}
& \text { If }\left(\left\{\bar{U}^{i}\right\}_{0}^{n},\left\{\bar{J}^{i}\right\}_{1}^{n}\right)=\mathcal{L}\left(\left\{U^{i}\right\}_{0}^{n},\left\{c^{i} \Delta^{i}\right\}_{1}^{n}\right), \\
& \text { then } \bar{U}^{i}(x)=\left(\left(D f^{i}-x I\right) U^{i}(x)\right)_{x}, \\
& \text { and } \bar{J}^{i}=\left(D f^{i}-x_{0}^{i} I\right) U^{i}\left(x_{0}^{i}\right)-\left(D f^{i-1}-x_{0}^{i} I\right) U^{i-1}\left(x_{0}^{i}\right) .
\end{aligned}
$$

Let $U_{\ell}^{R i}(x)=U_{\ell}^{R}(x), x \in R^{i}$. Then the eigenvalue problem can be recast into

$$
\begin{aligned}
& \left(\mathcal{L}+\left(\lambda_{0}+1\right) I\right)\left(\left\{U_{1}^{R i}\right\}_{0}^{n},\left\{c_{0}^{i} \Delta^{i}\right\}_{1}^{n}\right)=0 \\
& \left(\mathcal{L}+\left(\lambda_{0}+1\right) I\right)\left(\left\{U_{2}^{R i}\right\}_{0}^{n},\left\{c_{1}^{i} \Delta^{i}\right\}_{1}^{n}\right)+\lambda_{1}\left(\left\{U_{1}^{R i}\right\}_{0}^{n},\left\{c_{0}^{i} \Delta^{i}\right\}_{1}^{n}\right)=\left(\left\{W_{2}^{i}\right\}_{0}^{n},\left\{J_{2}^{i}\right\}_{1}^{n}\right) \\
& \left(\mathcal{L}+\left(\lambda_{0}+1\right) I\right)\left(\left\{U_{j}^{R i}\right\}_{0}^{n},\left\{c_{j-1}^{i} \Delta^{i}\right\}_{1}^{n}\right)+\lambda_{j-1}\left(\left\{U_{1}^{R i}\right\}_{0}^{n},\left\{c_{0}^{i} \Delta^{i}\right\}_{1}^{n}\right) \\
& =\left(\left\{W_{j}^{i}\right\}_{0}^{n},\left\{J_{j}^{i}\right\}_{1}^{n}\right)
\end{aligned}
$$

Here $\left(\left\{W_{j}^{i}\right\}_{0}^{n},\left\{J_{j}^{i}\right\}_{1}^{n}\right)$ as in (3.20), (3.23) denotes terms that involve indices lower than the $j$ th order. We assume that

(H1) The operator $\mathcal{L}$ has a simple eigenvalue $-\left(\lambda_{0}+1\right)$ with $\left(\left\{U_{1}^{R i}\right\}_{0}^{n},\left\{c_{0}^{i} \Delta^{i}\right\}_{1}^{n}\right)$ as an eigenvector.

We will show that from (H1) all the higher order expansions of the eigenvalue equation can be solved successively and $\lambda_{j-1}$ can be uniquely determined in the $\epsilon^{j}$ th expansion.

In the next subsection, we will show that $\mathcal{L}$ is Fredholm. We will introduce the SLEP matrix $\mathcal{M}(\lambda)$ and the SLEP function $p(\lambda)=\operatorname{det} \mathcal{M}(\lambda)$. We will show that (H1) is equivalent to that $p(\lambda)$ has a simple zero at $\lambda_{0}$.

4.1. Preliminaries. We now discuss properties of $\mathcal{L}+\left(\lambda_{0}+1\right) I$ and show that it is a Fredholm operator. Let $\Phi^{i}\left(y, x, \lambda_{0}\right)$ be the principal matrix solution for the system in $R^{i}$ :

$$
\left(\lambda_{0}+1\right) U+\left(\left(D f^{i}-x I\right) U\right)_{x}=0, \quad x \in R^{i} .
$$

Let $V=\left(D f^{i}-x I\right) U$, and $Q^{i}\left(y, x, \lambda_{0}\right)$ be the principal matrix solution for the associated system

$$
\left(\lambda_{0}+1\right)\left(D f^{i}-x I\right)^{-1} V+V_{x}=0 .
$$

Clearly, we have

$$
\Phi^{i}\left(y, x, \lambda_{0}\right)=\left(D f^{i}-y I\right)^{-1} Q^{i}\left(y, x, \lambda_{0}\right)\left(D f^{i}-x I\right) .
$$

Let $\Psi^{i}\left(x, y, \lambda_{0}\right)$ be the principal matrix solution for the adjoint system to (4.4).

$$
\left(\lambda_{0}^{*}+1\right) \psi-\left(D f^{i}-x I\right)^{*} \psi_{x}=0, \quad x \in R^{i} .
$$

It turns out that (4.6) is the adjoint system for both (4.4) and (4.5) in the sense that

$$
\frac{d}{d x}<\left(D f^{i}-x I\right) U, \psi>=\frac{d}{d x}<V, \psi>=0 .
$$

We have the following results: 
Lemma 4.1. For any $\xi \in R^{i}$ and $p \in C\left(R^{i}\right)$, the general solution for the nonhomogeneous equation in $R^{i}$

$$
\left(\lambda_{0}+1\right) U+\left(\left(D f^{i}-x I\right) U\right)_{x}=p(x)
$$

can be expressed as

$$
U(x)=\Phi^{i}\left(x, \xi, \lambda_{0}\right) U(\xi)+\int_{\xi}^{x} \Phi^{i}\left(x, \eta, \lambda_{0}\right)\left(D f^{i}-\eta I\right)^{-1} p(\eta) d \eta .
$$

For $x, y \in R^{i}$, we have

$$
\Psi^{i}\left(x, y, \lambda_{0}\right)=Q^{i *}\left(y, x, \lambda_{0}\right) .
$$

Moreover, $\Psi^{i}$ can be expressed by $\Phi^{i}$, vise-versa.

$$
\begin{aligned}
& \Psi^{i}\left(x, y, \lambda_{0}\right)=\left(D f^{i}-x I\right)^{-1, *} \Phi^{i *}\left(y, x, \lambda_{0}\right)\left(D f^{i}-y I\right)^{*}, \\
& \Phi^{i}\left(y, x, \lambda_{0}\right)=\left(D f^{i}-y I\right)^{-1} \Psi^{i *}\left(x, y, \lambda_{0}\right)\left(D f^{i}-x I\right) .
\end{aligned}
$$

The proof of Lemma 4.1 shall be omitted.

We will extend the domain of $Q^{i}$ and $\Psi^{i}$ to $\mathbb{R} \times \mathbb{R}$. Define

$$
Q\left(x_{0}^{i}, x_{0}^{j}, \lambda_{0}\right)=Q^{i-1}\left(x_{0}^{i}, x_{0}^{i-1} ; \lambda_{0}\right) \ldots Q^{j}\left(x_{0}^{j+1}, x_{0}^{j}, \lambda_{0}\right), \quad i>j .
$$

For $i<j$, let $Q\left(x_{0}^{i}, x_{0}^{j}, \lambda_{0}\right)=Q\left(x_{0}^{j}, x_{0}^{i}, \lambda_{0}\right)^{-1}$. Finally let $Q\left(x_{0}^{i}, x_{0}^{i}, \lambda_{0}\right)=I$. For $x_{0}^{i} \leq x \leq x_{0}^{i+1}, x_{0}^{j} \leq y \leq x_{0}^{j+1}$, let

$$
Q\left(y, x . \lambda_{0}\right)=Q^{j}\left(y, x_{0}^{j}, \lambda_{0}\right) Q\left(x_{0}^{j}, x_{0}^{i+1}, \lambda_{0}\right) Q^{i}\left(x_{0}^{i+1}, x . \lambda_{0}\right) .
$$

Finally, let

$$
\Psi\left(x, y, \lambda_{0}\right)=Q^{*}\left(y, x, \lambda_{0}\right) .
$$

Let $c^{i} \in \mathbb{R}, i=1, \ldots, n$ and $b^{i}, g^{i} \in \mathbb{R}^{n}, i=0, \ldots, n$. Motivated by (3.17), define

$$
\begin{aligned}
& G:\left(\left\{c^{i}\right\}_{1}^{n},\left\{b^{i}\right\}_{1}^{n-1}\right) \rightarrow\left\{g^{i}\right\}_{1}^{n}, \\
& g^{i}=\left(\lambda_{0}+1\right) c^{i} \Delta^{i}+\left(D f^{i}-x_{0}^{i}\right) b^{i}-\left(D f^{i-1}-x_{0}^{i}\right) \Phi^{i-1}\left(x_{0}^{i}, x_{0}^{i-1}, \lambda_{0}\right) b^{i-1} .
\end{aligned}
$$

For convenience, we assume that $b^{0}=b^{n}=0$. Since the domain and range of $G$ are both $n^{2}$-dimensional, $G$ is Fredholm with index 0 .

We have the following obvious lemma:

Lemma 4.2. $\left(\left\{U^{i}\right\}_{0}^{n},\left\{c^{i} \Delta^{i}\right\}_{1}^{n}\right)$ is an eigenfunction of $\mathcal{L}$ with respect to the eigenvalue $-\left(\lambda_{0}+1\right)$ if and only if

$$
\left(\left\{c^{i}\right\}_{1}^{n},\left\{U^{i}\left(x_{0}^{i}+\right)\right\}_{1}^{n-1}\right) \in \operatorname{ker}(G) .
$$

Let

$$
\mathcal{H}_{2}:=\left\{G\left(\{0\}_{1}^{n},\left\{b^{i}\right\}_{1}^{n-1}\right), \quad \text { for all } b^{i} \in \mathbb{R}^{n}\right\} .
$$

One can show that $\mathcal{H}_{2}$ consists of all the jumps coming from solutions of the homogeneous equation (4.4). The space $\mathcal{H}_{2}$ is $n(n-1)$ dimensional, i.e., with $c^{i} \equiv 0, G$ maps $\left\{b^{i}\right\}_{1}^{n-1}$ injectively into $\mathcal{H}$. This can be shown by using Schecter's condition on structural stability of Riemann solutions. (If the mapping from $\left\{b^{i}\right\}_{1}^{n-1}$ to the jumps were not one-to-one, then there would be a Riemann solution with zero Rankine-Hugoniot jump at each shock.) Observe that $\mathcal{H}_{1}=\left\{G\left(\left\{c^{i}\right\}_{1}^{n},\{0\}_{1}^{n-1}\right)\right.$ for all $\left.c^{i} \in \mathbb{R}\right\}$. It should be clear that the dimension of $\operatorname{ker}(G)$ is the dimension of $\mathcal{H}_{1} \cap \mathcal{H}_{2}$. 
We now introduce a matrix, similar to the SLEP matrix in Schecter \& Lin [19] and Nishiura \& Fujii [25] that helps to determine the kernel and co-kernel of $G$.

Assume that $G\left(\left\{c^{i}\right\}_{1}^{n},\left\{b^{i}\right\}_{1}^{n-1}\right)=0$. Define

$$
k^{i}=\left(D f^{i}-x_{0}^{i} I\right) b^{i}, \text { for } i=1, \ldots, n-1, \quad k^{0}=k^{n}=0 .
$$

Then from

$$
\left(D f^{i}-x_{0}^{i} I\right) b^{i}=\left(D f^{i-1}-x_{0}^{i}\right) \Phi^{i-1}\left(x^{i}, x_{0}^{i-1} ; \lambda_{0}\right) b^{i-1}-\left(\lambda_{0}+1\right) c^{i} \Delta^{i},
$$

we have

$$
k^{i}=Q^{i-1}\left(x_{0}^{i}, x_{0}^{i-1} ; \lambda_{0}\right) k^{i-1}-\left(\lambda_{0}+1\right) c^{i} \Delta^{i} .
$$

From (4.7),

$$
\begin{aligned}
& k^{i}=-\left(\lambda_{0}+1\right) \sum_{j=1}^{i} c^{j} Q\left(x_{0}^{i}, x_{0}^{j}, \lambda_{0}\right) \Delta^{j}, \\
& k^{n}=-\left(\lambda_{0}+1\right) \sum_{j=1}^{n} c^{j} Q\left(x_{0}^{n}, x_{0}^{j}, \lambda_{0}\right) \Delta^{j}=0 .
\end{aligned}
$$

Definition 4.1. (SLEP matrix and the SLEP function) The SLEP matrix $\mathcal{M}\left(\lambda_{0}\right)$ is the $n \times n$ matrix whose $j$ th column is the vector $Q\left(x_{0}^{n}, x_{0}^{j}, \lambda_{0}\right) \Delta^{j}$. The SLEP function $p(\lambda)=\operatorname{det} \mathcal{M}(\lambda)$.

Note that $k^{n}=0$ implies that $\left\{c^{i}\right\}_{1}^{n}$ is a right eigenvector of $\mathcal{M}\left(\lambda_{0}\right)$ :

$$
\mathcal{M}\left(\lambda_{0}\right)\left\{c^{i}\right\}_{1}^{n}=0, \quad \text { with } p\left(\lambda_{0}\right)=0 .
$$

Once $\left\{c^{i}\right\}_{1}^{n}$ is determined, $\left\{b^{i}\right\}_{1}^{n-1}$ can be calculated through $\left\{k^{i}\right\}_{1}^{n-1}$.

We now characterized the co-kernel of $G$. Let $\left\{d^{i}\right\}_{1}^{n} \in \mathcal{H}$ such that $\left\{d^{i}\right\}_{1}^{n} \perp$ range $G$.

$$
\sum_{1}^{n}\left(\lambda_{0}+1\right) c^{i}<d^{i}, \Delta^{i}>+\sum_{1}^{n}<d^{i},\left(D f^{i}-x_{0}^{i}\right) b^{i}-\left(D f^{i-1}-x_{0}^{i}\right) \Phi^{i-1}\left(x_{0}^{i}, x_{0}^{i-1} ; \lambda_{0}\right) b^{i-1}>=0 .
$$

The above is valid for all vectors $\left(\left\{c^{i}\right\}_{1}^{n},\left\{b^{i}\right\}_{1}^{n-1}\right)$. Recall we assume that $\lambda_{0}+1 \neq 0$. If we let $b^{i} \equiv 0$ for all $i$, then we have $<d^{i}, \Delta^{i}>=0, i=1, \ldots, n$. Consequently,

$$
\sum_{1}^{n}<d^{i},\left(D f^{i}-x_{0}^{i}\right) b^{i}-\left(D f^{i-1}-x_{0}^{i}\right) \Phi^{i-1}\left(x_{0}^{i}, x_{0}^{i-1}, \lambda_{0}\right) b^{i-1}>=0 .
$$

Therefore, $\left\{d^{i}\right\}_{1}^{n}$ is a vector in $\mathcal{H}$ determined by

$$
\left\{d^{i}\right\}_{1}^{n} \perp \mathcal{H}_{1}+\mathcal{H}_{2}
$$

Notice that the above also implies that the dimension of the linear space of such $\left\{d^{i}\right\}_{1}^{n}$ is the dimension of $\mathcal{H}_{1} \cap \mathcal{H}_{2}$ which is the dimension of $\operatorname{ker} G$.

Using $k^{i}=\left(D f^{i}-x_{0}^{i} I\right) b^{i}$, the conditions on $\left\{d^{i}\right\}_{1}^{n}$ can be expressed as

$$
\sum_{1}^{n}<d^{i}, k^{i}-Q^{i-1}\left(x_{0}^{i}, x_{0}^{i-1}, \lambda_{0}\right) k^{i-1}>\text {. }
$$


Recall that $Q^{i *}\left(x, y, \lambda_{0}\right)=\Psi^{i}\left(y, x, \lambda_{0}\right)$. Therefore,

$$
\sum_{1}^{n}<d^{i}-\Psi^{i}\left(x_{0}^{i}, x_{0}^{i+1}, \lambda_{0}\right) d^{i+1}, k^{i}>=0 .
$$

Since $k^{i}$ is arbitrary, we have the system that determines $\left\{d^{i}\right\}_{1}^{n}$ up to a scalar multiple:

$$
\begin{aligned}
& <d^{i}, \Delta^{i}>=0, \quad i=1, \ldots, n, \\
& d^{i}-\Psi^{i}\left(x_{0}^{i}, x_{0}^{i+1}, \lambda_{0}\right) d^{i+1}=0, \quad i=1, \ldots, i-1 .
\end{aligned}
$$

Then if $d^{n}$ is known, $d^{i}, i \leq n$ can be obtained from

$$
d^{i}=\Psi\left(x_{0}^{i}, x_{0}^{n} ; \lambda_{0}\right) d^{n}
$$

Since $<d^{i}, \Delta^{i}>=0, i=1, \ldots, n$, we have

$$
<d^{n}, Q\left(x_{0}^{n}, x_{0}^{i} ; \lambda_{0}\right) \Delta^{i}>=0, i=1, \ldots, n .
$$

This means that $d^{n}$ is a left eigenvector of the matrix $\mathcal{M}\left(\lambda_{0}\right)$. Once we have calculated $d^{n}$, the other vectors $d^{i}, i=1, \ldots, n-1$ can be obtained by using (4.8).

We summarize the results in the following lemma.

Lemma 4.3. $-\left(\lambda_{0}+1\right)$ is an eigenvalue of $\mathcal{L}$ iff $p\left(\lambda_{0}\right)=0$. Then $\left(\left\{c^{i}\right\}_{1}^{n},\left\{b^{i}\right\}_{1}^{n-1}\right)$ is in the kernel of $G$ iff

$$
\begin{aligned}
& \mathcal{M}\left(\lambda_{0}\right)\left\{c^{i}\right\}_{1}^{n}=0, \quad \text { with } p\left(\lambda_{0}\right)=0, \\
& b^{i}=-\left(\lambda_{0}+1\right)\left(D f^{i}-x_{0}^{i} I\right)^{-1} \sum_{j=1}^{i} c^{j} Q\left(x_{0}^{i}, x_{0}^{j}, \lambda_{0}\right) \Delta^{j} .
\end{aligned}
$$

Furthermore, $\left\{d^{i}\right\}_{1}^{n}$ is orthogonal to the range of $G$ iff

$$
\begin{aligned}
& \left(d^{n}\right)^{*} \mathcal{M}\left(\lambda_{0}\right)=0, \\
& d^{i}=\Psi\left(x_{0}^{i}, x_{0}^{n} ; \lambda_{0}\right) d^{n}, \quad i<n .
\end{aligned}
$$

Lemma 4.4. Assume that $p\left(\lambda_{0}\right)=0$. Then $\left(\left\{z^{i}(x)\right\}_{0}^{n},\left\{d^{i}\right\}_{1}^{n}\right)$ is orthogonal to the range of $\mathcal{L}+\left(\lambda_{0}+1\right) I$ if and only if

$$
\left\{d^{i}\right\}_{1}^{n} \perp \operatorname{Range}(G),
$$

and $\left\{z^{i}\right\}_{0}^{n}$ satisfies the adjoint equation (4.6)

$$
\left(\lambda_{0}^{*}+1\right) z^{i}-\left(D f^{i}-x I\right)^{*} z_{x}^{i}=0 \quad i=1, \ldots, n-1,
$$

with $z^{i}\left(x^{i+1}\right)=d^{i+1}$. Moreover, $z^{i}\left(x_{0}^{i}+\right)=z^{i-1}\left(x_{0}^{i}-\right)$.

Proof. If $<\left(\mathcal{L}+\left(\lambda_{0}+1\right) I\right)\left(\left\{U^{i}\right\}_{0}^{n},\left\{c^{i} \Delta^{i}\right\}_{1}^{n}\right),\left(\left\{z^{i}(x)\right\}_{0}^{n},\left\{d^{i}\right\}_{1}^{n}\right)>=0$ for all $\left(\left\{U^{i}\right\},\left\{c^{i}\right\}\right)$, then

$$
\begin{aligned}
0 & =\sum_{1}^{n-1} \int_{x^{i}}^{x^{i+1}}<\left(\lambda_{0}+1\right) U^{i}+\left(\left(D f^{i}-x I\right) U^{i}\right)_{x}, z^{i}>d x \\
& +\sum_{1}^{n}<\left(D f^{i}-x^{i} I\right) U^{i}\left(x^{i}\right)-\left(D f^{i-1}-x^{i} I\right) U^{i-1}\left(x^{i}\right)+\left(\lambda_{0}+1\right) c^{i} \Delta^{i}, d^{i}>.
\end{aligned}
$$


Let $U^{i} \equiv 0$. Then since $c^{i}$ is arbitrarily, we have

$$
<\Delta^{i}, d^{i}>=0, \quad i=1, \ldots, n .
$$

Integrating by parts, we have

$$
\begin{aligned}
0 & =\sum_{1}^{n-1}<\left(D f^{i}-x I\right) U^{i}(x), z^{i}(x)>\left.\right|_{x^{i}} ^{x^{i+1}} \\
& -\sum_{1}^{n-1} \int_{x^{i}}^{x^{i+1}}<U^{i},\left(\lambda_{0}^{*}+1\right) z^{i}-\left(D f^{i}-x I\right)^{*} z_{x}^{i}>d x \\
& +\sum_{1}^{n}<\left(D f^{i}-x^{i} I\right) U^{i}\left(x^{i}\right)-\left(D f^{i-1}-x^{i} I\right) U^{i-1}\left(x^{i}\right), d^{i}>.
\end{aligned}
$$

If $U^{i} \in C_{c}^{\infty}\left(x^{i}, x^{i+1}\right), i=1, \ldots, n-1$, then we find that $z^{i}$ must satisfies the adjoint equation (4.6):

$$
\left(\lambda_{0}^{*}+1\right) z^{i}-\left(D f^{i}-x I\right)^{*} z_{x}^{i}=0 \quad i=1, \ldots, n-1 .
$$

The boundary terms satisfy

$$
\begin{aligned}
0 & =\sum_{1}^{n-1}\left(<\left(D f^{i}-x^{i+1} I\right) U^{i}\left(x^{i+1}\right), z^{i}\left(x^{i+1}\right)>-<\left(D f^{i}-x^{i} I\right) U^{i}\left(x^{i}\right), z^{i}\left(x^{i}\right)>\right) \\
& +\sum_{1}^{n}<\left(D f^{i}-x^{i} I\right) U^{i}\left(x^{i}\right)-\left(D f^{i-1}-x^{i} I\right) U^{i-1}\left(x^{i}\right), d^{i}> \\
& =\sum_{1}^{n}<\left(D f^{i}-x^{i} I\right) U^{i}\left(x^{i}\right),\left(d^{i}-z^{i}\left(x^{i}\right)\right)> \\
& -\sum_{1}^{n}<\left(D f^{i-1}-x^{i} I\right) U^{i-1}\left(x^{i}\right),\left(d^{i}-z^{i-1}\left(x^{i}\right)\right)>.
\end{aligned}
$$

Since $U^{i}\left(x^{i}\right)$ and $U^{i-1}\left(x^{i}\right)$ can be arbitrary constants, we have

$$
z^{i-1}\left(x^{i}\right)=z^{i}\left(x^{i}\right)=d^{i}, \quad i=1, \ldots, n .
$$

Define $z(x)=z^{i}(x)$ for $x \in\left[x^{i}, x^{i+1}\right]$. Then $z$ is continuous on $\left[x_{0}^{1}, x_{0}^{n}\right]$.

$$
z\left(x^{i}\right)=d^{i}=\Psi\left(x^{i}, x^{n}, \lambda_{0}\right) d^{n} .
$$

Using $<\Delta^{i}, d^{i}>=0$ for all $i$, and $\Psi^{*}=Q$, we have

$$
\begin{aligned}
& <\Delta^{i}, \Psi\left(x^{i}, x^{n}, \lambda_{0}\right) d^{n}>=0, \\
& <Q\left(x^{n}, x^{i}, \lambda_{0}\right) \Delta^{i}, d^{n}>=0 .
\end{aligned}
$$

By the definition of $M\left(\lambda_{0}\right)$, we have

$$
d^{n *} M\left(\lambda_{0}\right)=0
$$

That is, $d^{n}$ is a left eigenvector for the matrix $M\left(\lambda_{0}\right)$. Based on Lemma 4.3, it follows from (4.10) and (4.9) that $\left\{d^{i}\right\}_{1}^{n}$ is orthogonal to the range of $G$. 
Definition 4.2. Let $\mathcal{K}\left(\lambda_{0}\right)$ be the linear space of continuous functions defined on $\left[x_{0}^{1}, x_{0}^{n}\right]$ such that if $z \in \mathcal{K}\left(\lambda_{0}\right)$ then

(1) $z$ is piecewise continuously differentiable and satisfies the adjoint equation (4.6) on $R^{i}, i=1, \ldots, n-1$;

(2) $\left\{z\left(x_{0}^{i}\right)\right\}_{1}^{n}$ is orthogonal to the range of $G$.

From the definition, it is clear that $z \in \mathcal{K}\left(\lambda_{0}\right)$ if and only if

$$
\begin{aligned}
& z^{*}\left(x_{0}^{n}\right) \mathcal{M}\left(\lambda_{0}\right)=0, \\
& z(x)=\Psi\left(x, x_{0}^{n}, \lambda_{0}\right) z\left(x_{0}^{n}\right) .
\end{aligned}
$$

We can state the basic properties of $\mathcal{L}+\left(\lambda_{0}+1\right) I$ as follows:

Theorem 4.5. $-\left(\lambda_{0}+1\right)$ is an eigenvalue for the operator $\mathcal{L}$ iff $p\left(\lambda_{0}\right)=0$. Then $\mathcal{L}+\left(\lambda_{0}+1\right) I$ is Fredholm with the index zero. The condition for $\left(\left\{U^{i}\right\}_{0}^{n},\left\{c^{i} \Delta^{i}\right\}_{1}^{n}\right)$ being an eigenvector is

$$
\begin{aligned}
& \mathcal{M}\left(\lambda_{0}\right)\left\{c^{i}\right\}_{1}^{n}=0, \\
& U^{i}\left(x_{0}^{i}\right)=-\left(\lambda_{0}+1\right)\left(D f^{i}-x_{0}^{i} I\right)^{-1} \sum_{j=1}^{i} c^{j} Q\left(x_{0}^{i}, x_{0}^{j}, \lambda_{0}\right) \Delta^{j}, \\
& U^{i}(x)=\Phi^{i}\left(x, x_{0}^{i}, \lambda_{0}\right) U^{i}\left(x_{0}^{i}\right), \quad x_{0}^{i} \leq x \leq x_{0}^{i+1} .
\end{aligned}
$$

Furthermore, $\left(\left\{W^{i}\right\}_{0}^{n},\left\{J^{i}\right\}_{1}^{n}\right) \in \mathcal{R}\left(\mathcal{L}+\left(\lambda_{0}+1\right) I\right)$ if and only if for all $z \in \mathcal{K}\left(\lambda_{0}\right)$,

$$
\sum_{1}^{n}<z\left(x_{0}^{i}\right), J^{i}>+\sum_{1}^{n-1} \int_{x_{0}^{i}}^{x_{0}^{i+1}}<z(x), W^{i}(x)>d x=0 .
$$

Proof. The kernel part of the theorem follows from Lemma 4.2 and Lemma 4.3. If $\left(\left\{W^{i}\right\}_{0}^{n},\left\{J^{i}\right\}_{1}^{n}\right) \in \mathcal{R}\left(\mathcal{L}+\left(\lambda_{0}+1\right) I\right)$, then

$$
\begin{aligned}
& \left(\lambda_{0}+1\right) U^{i}+\left(\left(D f^{i}-x I\right) U^{i}\right)_{x}=W^{i}, \\
& \left(\lambda_{0}+1\right) c^{i} \Delta^{i}+\left(D f^{i}-x_{0}^{i} I\right) U^{i}\left(x_{0}^{i}\right)-\left(D f^{i-1}-x_{0}^{i} I\right) U^{i-1}\left(x_{0}^{i}\right)=J^{i} .
\end{aligned}
$$

Let $b^{i}=U^{i}\left(x_{0}^{i}\right)$. Then

$$
U^{i}(x)=\Phi^{i}\left(x, x_{0}^{i}\right) b^{i}+\int_{x_{0}^{i}}^{x} \Phi^{i}(x, \eta)\left(D f^{i}-\eta I\right)^{-1} W^{i}(\eta) d \eta .
$$

Plug into (4.12), we have

$$
\begin{aligned}
\left(\lambda_{0}+1\right) c^{i} \Delta^{i}+\left(D f^{i}-x_{0}^{i} I\right) b^{i}-\left(D f^{i-1}-x_{0}^{i} I\right) \Phi^{i-1}\left(x_{0}^{i}, x_{0}^{i-1}\right) b^{i-1} \\
=\left(D f^{i-1}-x_{0}^{i} I\right) \int_{x_{0}^{i-1}}^{x_{0}^{i}} \Phi^{i-1}\left(x_{0}^{i}, \eta\right)\left(D f^{i-1}-\eta I\right)^{-1} W^{i-1}(\eta) d \eta+J^{i}
\end{aligned}
$$


The above has a solution iff the right hand side of (4.13) is in the range of $G$, i.e., for each $\left\{d^{i}\right\}_{1}^{n}$ that is orthogonal to the range of $G$ as in Lemma 4.3, we have

$$
\begin{aligned}
0 & =\sum_{1}^{n}<d^{i}, J^{i}+\left(D f^{i-1}-x_{0}^{i} I\right) \int_{x_{0}^{i-1}}^{x_{0}^{i}} \Phi^{i-1}\left(x_{0}^{i}, \eta\right)\left(D f^{i-1}-\eta I\right)^{-1} W^{i-1}(\eta) d \eta> \\
& =\sum_{1}^{n}<d^{i}, J^{i}> \\
& +\sum_{1}^{n} \int_{x_{0}^{i-1}}^{x_{0}^{i}}<\left(D f^{i-1}-\eta I\right)^{-1, *} \Phi^{i-1, *}\left(x_{0}^{i}, \eta, \lambda_{0}\right)\left(D f^{i-1}-x_{0}^{i} I\right)^{*} d^{i}, W^{i-1}(\eta)>d \eta
\end{aligned}
$$

Define

$$
\begin{gathered}
z^{i}(\eta)=\left(D f^{i}-\eta I\right)^{-1, *} \Phi^{i *}\left(x_{0}^{i+1}, \eta, \lambda_{0}\right)\left(D f^{i}-x_{0}^{i+1} I\right)^{*} d^{i+1}, \quad x_{0}^{i} \leq \eta \leq x_{0}^{i+1} \\
=\Psi^{i}\left(\eta, x^{i+1}, \lambda_{0}\right) d^{i+1} .
\end{gathered}
$$

Then

$$
\begin{aligned}
0 & =\sum_{1}^{n}<d^{i}, J^{i}>+\sum_{1}^{n} \int_{x_{0}^{i-1}}^{x_{0}^{i}}<z^{i-1}(\eta), W^{i-1}(\eta)>d \eta \\
& =\sum_{1}^{n}<d^{i}, J^{i}>+\sum_{1}^{n-1} \int_{x_{0}^{i}}^{x_{0}^{i+1}}<z^{i}(\eta), W^{i}(\eta)>d \eta .
\end{aligned}
$$

The functions $\left\{z^{i}\right\}_{1}^{n-1}$ can be glued together to be a continuous function $z$ defined on $\left[x_{0}^{1}, x_{0}^{n}\right]$. We clearly have $z \in \mathcal{K}\left(\lambda_{0}\right)$.

The following lemmas characterizes the condition that $-\left(\lambda_{0}+1\right)$ is a simple eigenvalue of $\mathcal{L}$.

Lemma 4.6. The eigenvalue $-\left(\lambda_{0}+1\right)$ of $\mathcal{L}$ is semisimple if and only if for any eigenfunction $\left(\left\{U_{1}^{R i}(x)\right\}_{0}^{n},\left\{c_{0}^{i}\right\}_{1}^{n}\right)$, there exists at least one $z \in \mathcal{K}\left(\lambda_{0}\right)$ such that

$$
\sum_{1}^{n-1} \int_{x_{0}^{i}}^{x_{0}^{i+1}}<z(x), U_{1}^{R i}(x)>d x \neq 0
$$

Proof. If there exists one $z \in \mathcal{K}\left(\lambda_{0}\right)$ such that

$$
0 \neq \sum_{1}^{n}<z\left(x_{0}^{i}\right), c_{0}^{i} \Delta^{i}>+\sum_{1}^{n-1} \int_{x_{0}^{i}}^{x_{0}^{i+1}}<z(x), U_{1}^{R i}(x)>d x
$$

then $\left(\left\{U_{1}^{R i}(x)\right\}_{0}^{n},\left\{c_{0}^{i}\right\}_{1}^{n}\right)$ is not on the range of $\mathcal{L}+\left(\lambda_{0}+1\right) I$. Since $<z\left(x_{0}^{i}\right), \Delta^{i}>=0$ for all $i$, the desired result follows.

Lemma 4.7. The condition that $-\left(\lambda_{0}+1\right)$ is a simple eigenvalue of $\mathcal{L}$ is equivalent to that the SLEP function $p(\lambda)=\operatorname{det} \mathcal{M}(\lambda)$ has a simple zero at $\lambda=\lambda_{0}$. 
Proof. Let the $i$ th column of $\mathcal{M}(\lambda)$ be $\mathcal{M}_{i}(\lambda)$.

$$
\mathcal{M}(\lambda)=\left(\mathcal{M}_{1}(\lambda), \mathcal{M}_{2}(\lambda), \ldots, \mathcal{M}_{n}(\lambda)\right) .
$$

Without loss of generality, let $\lambda_{0}=0$. We assume that the geometric multiplicity of $\lambda=0$ for the matrix $\mathcal{M}(0)$ is one since this is the consequence of either $p^{\prime}(0) \neq 0$ or $-\left(\lambda_{0}+1\right)$ is a simple eigenvalue of $\mathcal{L}$.

Let $\left\{c^{i}\right\}_{i=1}^{n},\left\{e_{j}\right\}_{j=1}^{n}$ be the right and left eigenvectors of $\mathcal{M}(0)$, unique up to scalar multipliers. Without loss of generality, we assume that $c^{1}=1$. Then $\mathcal{M}_{j}(0), j=$ $2, \ldots, n$ are linearly independent and

$$
\left\{e_{j}\right\} \perp \operatorname{span}\left\{\mathcal{M}_{j}(0)\right\}_{j=2}^{n} .
$$

We have the following decomposition

$$
\mathcal{M}(\lambda)\left\{c^{i}\right\}=\alpha(\lambda)\left\{e_{j}\right\}+\sum_{j=2}^{n} \beta_{j}(\lambda) \mathcal{M}_{j}(0) .
$$

Since the left hand side is $O(\lambda)$ we have

$$
\begin{gathered}
\alpha(\lambda)=O(\lambda), \quad \beta_{j}(\lambda)=O(\lambda), j=2, \ldots, n \\
\operatorname{det} \mathcal{M}(\lambda)=\operatorname{det}\left(\mathcal{M}(\lambda)\left\{c^{i}\right\}, \mathcal{M}_{2}(\lambda), \ldots, \mathcal{M}_{n}(\lambda)\right) \\
=\operatorname{det}\left(\alpha(\lambda)\left\{e_{j}\right\}+\sum_{j=2}^{n} \beta_{j}(\lambda) \mathcal{M}_{j}(0), \mathcal{M}_{2}(\lambda), \ldots, \mathcal{M}_{n}(\lambda)\right) .
\end{gathered}
$$

Since the first column is of $O(\lambda)$, if we replace $\mathcal{M}_{j}(\lambda)$ by $\mathcal{M}_{j}(0)$, we have:

$$
\begin{gathered}
p(\lambda)=O\left(\lambda^{2}\right)+\operatorname{det}\left(\alpha(\lambda)\left\{e_{j}\right\}+\sum_{j=2}^{n} \beta_{j}(\lambda) \mathcal{M}_{j}(0), \mathcal{M}_{2}(0), \ldots, \mathcal{M}_{n}(0)\right) \\
=O\left(\lambda^{2}\right)+\operatorname{det}\left(\alpha(\lambda)\left\{e_{j}\right\}, \mathcal{M}_{2}(0), \ldots, \mathcal{M}_{n}(0)\right) \\
=O\left(\lambda^{2}\right)+\alpha(\lambda) \operatorname{det}\left(\left\{e_{j}\right\}, \mathcal{M}_{2}(0), \ldots, \mathcal{M}_{n}(0)\right) . \\
\frac{d}{d \lambda} p(0)=\alpha^{\prime}(0) \operatorname{det}\left(\left\{e_{j}\right\}, \mathcal{M}_{2}(0), \ldots, \mathcal{M}_{n}(0)\right) .
\end{gathered}
$$

Assume that $\sum_{j}\left|e_{j}\right|^{2}=1$. Then

$$
\alpha(\lambda)=\left\{e_{j}\right\}^{*} \mathcal{M}(\lambda)\left\{c^{i}\right\} .
$$

Differentiating and setting $\lambda=0$, we have

$$
\frac{d}{d \lambda} \alpha(0)=\left\{e_{j}\right\}^{*} \frac{d}{d \lambda} \mathcal{M}(0)\left\{c^{i}\right\}
$$

Let $V(x)=\partial_{\lambda} Q\left(x, x_{0}^{i}, 0\right) \Delta^{i}$. Then

$$
V^{\prime}+\left(\lambda_{0}+1\right)(D f-x I)^{-1} V+(D f-x I)^{-1} Q\left(x, x_{0}^{i}, 0\right) \Delta^{i}=0,
$$

with the initial condition $V\left(x_{0}^{i}\right)=0$. Thus

$$
V\left(x_{0}^{n}\right)=-\int_{x_{0}^{i}}^{x_{0}^{n}} Q\left(x_{0}^{n}, y, 0\right)(D f-y I)^{-1} Q\left(y, x_{0}^{i}, 0\right) \Delta^{i} d y .
$$


From (4.14), and using Theorem 4.5 for $U_{1}^{R i}(x)$, we have

$$
\begin{array}{r}
-\frac{d}{d \lambda} \alpha(0)=\sum_{i=1}^{n}<\left\{e_{j}\right\}, \int_{x_{0}^{i}}^{x_{0}^{n}} Q\left(x_{0}^{n}, y, 0\right)(D f-y I)^{-1} Q\left(y, x_{0}^{i}, 0\right) \Delta^{i} c^{i}>d y \\
=\sum_{i=1}^{n-1}<\Psi\left(y, x_{0}^{n}, 0\right)\left\{e_{j}\right\}, \int_{x_{0}^{i}}^{x_{0}^{n}}(D f-y I)^{-1} Q\left(y, x_{0}^{i}, 0\right) \Delta^{i} c^{i}>d y \\
=\sum_{i=1}^{n-1} \int_{x_{0}^{i}}^{x_{0}^{i+1}}<z(y), \sum_{j=1}^{i}(D f-y I)^{-1} Q\left(y, x_{0}^{j}, 0\right) \Delta^{j} c^{j}>d y \\
=-\left(\lambda_{0}+1\right)^{-1} \sum_{1}^{n-1} \int_{x_{0}^{i}}^{x_{0}^{i+1}}<z(x), U_{1}^{R i}(x)>d x
\end{array}
$$

Here $z \in \mathcal{K}(0)$. The final result follows from Lemma 4.6.

4.2. Solving the SLEP recursively. Assume that $\lambda_{0}$ is a simple root for the SLEP function $p(\lambda)$. From Lemma 4.7, Hypothesis (H1) is satisfied. To solve (4.1), we let $\left\{c_{0}^{i}\right\}_{1}^{n}$ be a right eigenvector of $\mathcal{M}\left(\lambda_{0}\right)$. Then from Theorem 4.5,

$$
\begin{gathered}
U_{1}^{R i}\left(x_{0}^{i}\right)=-\left(\lambda_{0}+1\right)\left(D f^{i}-x_{0}^{i} I\right)^{-1} \sum_{j=1}^{i} c_{0}^{j} Q\left(x_{0}^{i}, x_{0}^{i}, \lambda_{0}\right) \Delta^{j}, \\
U_{1}^{R i}(x)=Q\left(x, x_{0}^{i}, \lambda_{0}\right) U_{1}^{R i}\left(x_{0}^{i}\right) .
\end{gathered}
$$

The kernel of $\mathcal{M}\left(\lambda_{0}\right)$ is one-dimensional. Therefore, $\mathcal{K}\left(\lambda_{0}\right)$ is also one-dimensional.

We then proceed by induction. If (4.3) has been solved for $j \leq k-1$, then to solve (4.3) for $j=k$, we need to select $\lambda_{k-1}$ so that

$$
\lambda_{k-1}\left(\left\{U_{1}^{R i}\right\}_{0}^{n},\left\{c_{0}^{i} \Delta^{i}\right\}_{1}^{n}\right)-\left(\left\{W_{k-1}^{i}\right\}_{0}^{n},\left\{J_{k-1}^{i}\right\}_{1}^{n}\right)
$$

is in the range of $\mathcal{L}+\left(\lambda_{0}+1\right)$. Let $z$ be a nonzero vector in $\mathcal{K}\left(\lambda_{0}\right)$. From Theorem 4.5,

$$
\begin{aligned}
\lambda_{k-1} \int_{x_{0}^{1}}^{x_{0}^{n}}<z(x), U_{1}^{R}(x)>d x & \\
& \quad-\sum_{1}^{n}<z\left(x_{0}^{i}\right), J_{k-1}^{i}>-\sum_{1}^{n-1} \int_{x_{0}^{i}}^{x_{0}^{i+1}}<z(x), W_{k-1}^{i}(x)>d x=0 .
\end{aligned}
$$

Since $-\left(\lambda_{0}+1\right)$ is a simple eigenvalue, based on Lemma 4.6, we have

$$
\int_{x_{0}^{1}}^{x_{0}^{n}}<z(x), U_{1}^{R}(x)>d x \neq 0 .
$$

Thus, $\lambda_{k-1}$ can be solved from (4.15). With this $\lambda_{k-1}$, there exists a nonunique $\left(\left\{U_{k}^{R i}\right\}_{0}^{n},\left\{c_{k-1}^{i} \Delta^{i}\right\}_{1}^{n}\right)$.

To uniquely determine $\left(\left\{U_{k}^{R i}\right\}_{0}^{n},\left\{c_{k-1}^{i} \Delta^{i}\right\}_{1}^{n}\right)$, we assume that

$$
\sum_{1}^{n} c_{k-1}^{i} c_{0}^{i}=0 \text {. }
$$


This is a unnatural restriction on eigenfunctions. Multiplying an arbitrary analytic function $\alpha(\epsilon)$ to the eigenfunction, we recover all the eigenfunctions associated to the eigenvalue $-\left(\lambda_{0}+1\right)$.

\section{Existence of tRUe EIGENVALUE/EIGENFUnCtions}

Assume that we have the expansion of the slow eigenvalue up to $\epsilon^{m-1}$, and the associated eigenfunction up to $\epsilon^{m}, m \geq 1$. Let $0<\beta<1$ be an arbitrary constant. Define the inner layer $I_{\epsilon}^{i}:=\left\{x \mid x_{0}^{i}-\epsilon^{\beta} \leq x \leq x_{0}^{i}+\epsilon^{\beta}\right\}$ and the outer layer $O_{\epsilon}^{i}:=$ $\left\{x \mid x_{0}^{i}+\epsilon^{\beta} \leq x \leq x_{0}^{i+1}-\epsilon^{\beta}\right\}$. For brevity, let $a^{i}=x_{0}^{i}+\epsilon^{\beta}, b^{i}=x_{0}^{i+1}-\epsilon^{\beta}$ so that $O_{\epsilon}^{i}=\left[a^{i}, b^{i}\right]$. In the classical singular perturbation theory, $\epsilon^{\beta}$ is so called an intermediate variable $[21,10]$. Note that in the $x$ variable, the length of inner layers goes to zero as $\epsilon \rightarrow 0$, but in the stretched variable $\xi=\left(x-x_{0}^{i}\right) / \epsilon$, the length $O\left(\epsilon^{\beta-1}\right)$ goes to infinity as $\epsilon \rightarrow 0$. Therefore, the dynamics in both inner and outer layers are dominated by the exponential dichotomies using the variable $\xi$.

Define the approximation of eigenvalue/eigenfunctions by

$$
\begin{aligned}
& \lambda_{a p}=\sum_{0}^{m-1} \epsilon^{j} \lambda_{j}, \quad m \geq 1, \\
& U_{a p}^{R}=\sum_{0}^{m} \epsilon^{j} U_{j}^{R}, \quad \text { for all } x \in O_{\epsilon}^{i}, \\
& U_{a p}^{i}=\sum_{0}^{m} \epsilon^{j} U_{j}^{i}, \quad-\epsilon^{\beta-1} \leq \xi \leq \epsilon^{\beta-1}, x_{0}^{i}+\epsilon \xi=x \in I_{\epsilon}^{i} .
\end{aligned}
$$

Notice that $U_{0}^{i}=q_{\xi}^{i}(\xi), U_{0}^{R} \equiv 0$. In the expression $U_{j}^{i}=U_{j}^{i \perp}+c_{j}^{i} q_{\xi}^{i}$, the terms $U_{j}^{i \perp}, j \leq m$, and $c_{j}^{i}, j \leq m-1$, are determined while $c_{m}^{i}$ is still undetermined. Without loss of generality, let $c_{m}^{i}=0$.

Gluing the approximations in outer and inner layers together in the order of

$$
O_{\epsilon}^{0}, I_{\epsilon}^{1}, O_{\epsilon}^{1}, I_{\epsilon}^{2}, \ldots, I_{\epsilon}^{n}, O_{\epsilon}^{n},
$$

we have a so called pseudo orbit with small residual and jump errors:

$$
\begin{aligned}
& \left(\lambda_{a p}+1\right) U_{a p}^{R}+\left(\left(D f\left(u_{\epsilon}\right)-x I\right) U_{a p}^{R}\right)_{x}-\epsilon U_{a p, x x}^{R}=\epsilon h^{R}, \quad \text { in } O_{\epsilon}^{i}, \\
& \epsilon\left(\lambda_{a p}+1\right) U_{a p}^{i}+\left(\left(D f\left(u_{\epsilon}\right)-x^{i}(\epsilon)-\epsilon \xi\right) U_{a p}^{i}\right)_{\xi}-U_{a p, \xi \xi}^{i}=\epsilon h^{i}, \quad \text { in } I_{\epsilon}^{i}, \\
& U_{a p}^{i}\left(-\epsilon^{\beta-1}\right)-U_{a p}^{R}\left(x_{0}^{i}-\epsilon^{\beta}\right)=-\delta U^{i,-}, \\
& U_{a p, \xi}^{i}\left(-\epsilon^{\beta-1}\right)-\epsilon U_{a p, x}^{R}\left(x_{0}^{i}-\epsilon^{\beta}\right)=-\delta U_{\xi}^{i,-}, \\
& U_{a p}^{R}\left(x_{0}^{i}+\epsilon^{\beta}\right)-U_{a p}^{i}\left(\epsilon^{\beta-1}\right)=-\delta U^{i,+}, \\
& \epsilon U_{a p, x}^{R}\left(x_{0}^{i}+\epsilon^{\beta}\right)-U_{a p, \xi}^{i}\left(\epsilon^{\beta-1}\right)=-\delta U_{\xi}^{i,+} .
\end{aligned}
$$

The residual and jump errors satisfy

$$
\begin{aligned}
& \epsilon h^{R}=O\left(\epsilon^{m+1}\right), \epsilon h^{i}=O\left(\epsilon^{m+1}|\xi|^{m+1}\right)=O\left(\epsilon^{\beta(m+1)}\right) \\
& \left|\delta U^{i, \pm}\right|+\left|\delta U_{\xi}^{i, \pm}\right| \leq C e^{-\alpha \epsilon^{\beta-1}}\left(\epsilon^{\beta-1}\right)^{m}
\end{aligned}
$$


If $m \geq 1$ and $1 / 2<\beta<1$, then

$$
h^{R}=O\left(\epsilon^{m}\right), h^{i}=O\left(\epsilon^{2 \beta-1+\beta(m-1)}\right) \rightarrow 0, \quad \epsilon \rightarrow 0 .
$$

The exact eigenvalue/eigenfunctions satisfy

$$
\begin{array}{r}
\left(\lambda_{e x}+1\right) U_{e x}^{R}+\left(\left(D f\left(u_{\epsilon}\right)-x I\right) U_{e x}^{R}\right)_{x}=\epsilon U_{e x, x x}^{R}, \\
\epsilon\left(\lambda_{e x}+1\right) U_{e x}^{i}+\left(\left(D f\left(u_{\epsilon}\right)-x^{i}(\epsilon)-\epsilon \xi\right) U_{e x}^{i}\right)_{\xi}=U_{e x, \xi \xi}^{i} .
\end{array}
$$

Write the exact eigenvalue/eigenfunctions as approximations plus correction terms:

$$
\begin{aligned}
\lambda_{e x} & =\lambda_{a p}+\lambda, \\
U_{e x}^{R} & =U_{a p}^{R}+\epsilon U^{R}, \quad x \in O_{\epsilon}^{i}, \\
U_{e x}^{i} & =U_{a p}^{i}+\epsilon U^{i}+\epsilon^{m} c^{i} q_{\xi}^{i}(\xi), \quad x \in I_{\epsilon}^{i} .
\end{aligned}
$$

We easily find that the equations for $\left(\lambda, U^{R}, U^{i}\right)$ can be written as a linear variational system with forcing terms:

$$
\begin{aligned}
\left(\lambda_{0}+1\right) U^{R}+\lambda U_{1}^{R} & \left.+\left(D f\left(u_{0}^{R}\right)-x I\right) U^{R}\right)_{x}-\epsilon U_{x x}^{R}=-h^{R}+N^{R}\left(U^{R}, \lambda, \epsilon\right), \\
\left(\lambda_{0}+1\right) c^{i} q_{\xi}^{i}(\xi)+\lambda U_{0}^{i}+ & {\left[\left(D^{2} f\left(q^{i}\right) u_{1}^{i}-\left(x_{1}^{i}+\xi\right) I\right) c^{i} q_{\xi}^{i}\right]_{\xi} } \\
+ & \left.\left(D f\left(q^{i}\right)-x_{0}^{i} I\right) U^{i}\right)_{\xi}-U_{\xi \xi}^{i}=-h^{i}+N^{i}\left(U^{i}, \lambda, c^{i}, \epsilon\right) .
\end{aligned}
$$

Equation (5.2) is valid in outer layers $O_{\epsilon}^{i}, 0 \leq i \leq n$ while (5.3) is valid in inner layers $I_{\epsilon}^{i}, 1 \leq i \leq n$. The nonlinear terms $N^{R}, N^{i}$ are small if $\left(U^{R}, U^{i}, \lambda, c^{i}, \epsilon\right)$ are small.

$$
\begin{aligned}
N^{R}\left(U^{R}, \lambda, \epsilon\right) & =O\left(\epsilon\left|U^{R}\right|_{C^{1}}+\epsilon|\lambda|\right), \\
N^{i}\left(U^{i}, \lambda, c^{i}, \epsilon\right) & =O\left(\epsilon^{\beta}\left|U^{i}\right|_{C^{1}}+\epsilon|\lambda|+\epsilon\left|c^{i}\right|+\left|c^{i}\right||\lambda|\right) .
\end{aligned}
$$

Jump conditions that cancel the jump errors of approximations between two adjacent inner and outer layers are prescribed:

$$
\begin{aligned}
& U^{i}\left(-\epsilon^{\beta-1}\right)-U^{R}\left(x_{0}^{i}-\epsilon^{\beta}\right)=\delta U^{i,-}, \\
& U_{\xi}^{i}\left(-\epsilon^{\beta-1}\right)-\epsilon U_{x}^{R}\left(x_{0}^{i}-\epsilon^{\beta}\right)=\delta U_{\xi}^{i,-}, \\
& U^{R}\left(x_{0}^{i}+\epsilon^{\beta}\right)-U^{i}\left(\epsilon^{\beta-1}\right)=\delta U^{i,+}, \\
& \epsilon U_{x}^{R}\left(x_{0}^{i}+\epsilon^{\beta}\right)-U_{\xi}^{i}\left(\epsilon^{\beta-1}\right)=\delta U_{\xi}^{i,+} .
\end{aligned}
$$

As before, boundary conditions for $x \rightarrow \pm \infty$ must be satisfied:

$$
\left(U, U_{x}\right)=O\left(e^{-\gamma|x|}\right), \quad x \in O_{\epsilon}^{0} \cup O_{\epsilon}^{n}
$$

In Theorem 5.7, the nonlinear system (5.2)-(5.5) will be solved by the contraction mapping principle. To this end, we consider the following linear non-homogeneous system with the same jump and boundary conditions (5.4) and (5.5):

$$
\begin{aligned}
\left(\lambda_{0}+1\right) U^{R}+\lambda U_{1}^{R} & \left.+\left(D f\left(u_{0}^{R}\right)-x I\right) U^{R}\right)_{x}-\epsilon U_{x x}^{R}=-h^{R}(x), \\
\left(\lambda_{0}+1\right) c^{i} q_{\xi}^{i}(\xi)+\lambda U_{0}^{i} & +\left[\left(D^{2} f\left(q^{i}\right) u_{1}^{i}-\left(x_{1}^{i}+\xi\right) I\right) c^{i} q_{\xi}^{i}\right]_{\xi} \\
+ & \left.\left(D f\left(q^{i}\right)-x_{0}^{i} I\right) U^{i}\right)_{\xi}-U_{\xi \xi}^{i}=-h^{i} .
\end{aligned}
$$

The usual way of writing (5.6) as first order systems, i.e.,

$$
U_{x}=V, \quad V_{x}=\ldots,
$$


does not work well in our case because the variables $(U, V)$ do not capture the fastslow behavior of the singularly perturbed problem. If a system is autonomous, following the general instruction of [13], §1.6, the slow variable should correspond to eigenspaces of zero eigenvalues while the fast variable should correspond to eigenspaces of nonzero eigenvalues. If we write our equation in outer layers using $\xi=(x-\bar{x}) / \epsilon, \bar{x} \in$ $O_{\epsilon}^{i}$, the homogeneous part of the equation becomes

$$
\left.\left(D f\left(u_{0}^{R}\right)-(\bar{x}+\epsilon \xi) I\right) U^{R}\right)_{\xi}-U_{\xi \xi}^{R}=0 .
$$

We find that the system is slow varying and can be approximated by an autonomous system. If we free $x=\bar{x}$ in the coefficients by letting $\epsilon=0$, the above system has $n$ zero eigenvalues and $n$ eigenvalues with nonzero real parts. If $\epsilon$ is small, the slow varying equation also admits a splitting of center subspace of dimension $n$ and and a hyperbolic subspace of dimension $n$. This wonderful theorem in a more general form can be found in [5] and can also be proved by geometric singular perturbation theory after adding equations $\dot{x}=\epsilon, \dot{\epsilon}=0$ to the system.

By calculating eigenvalues and eigenvectors for $\epsilon=0$. We find that the eigenspace that corresponds to eigenvalues with nonzero real parts is spanned by $\left(U, U_{\xi}\right)=$ $\left(\mathbf{r}_{j}, \nu_{j} \mathbf{r}_{j}\right)$, any $\left(U, U_{\xi}\right)$ in such space must satisfy $\epsilon U_{x}-\left(D f\left(u_{0}^{R}\right)-x I\right) U=U_{\xi}-$ $\left(D f\left(u_{0}^{R}\right)-x I\right) U=0$. We thus introduce a new variable

$$
V^{R}:=\epsilon U_{x}^{R}-\left(D f\left(u_{0}^{R}\right)-x I\right) U^{R}=U_{\xi}^{R}-\left(D f\left(u_{0}^{R}\right)-x I\right) U^{R}, \quad x \in O_{\epsilon}^{i} .
$$

For simplicity, the symbol $D f$ or $D f^{i}$ represents $D f\left(u_{0}^{R}\right)$ (constant matrix) in outer layers $O_{\epsilon}^{i}$ and $D f\left(q^{i}(\xi)\right)$ in inner layers $I_{\epsilon}^{i}$. When $\epsilon=0$, the eigenspace that corresponds to zero eigenvalues is $\left(U, U_{\xi}\right)=(U, 0)$. If we make the change of variable

$$
\Delta U^{R}=U^{R}+(D f-x I)^{-1} V^{R}=(D f-x I)^{-1} U_{\xi},
$$

then the eigenspaces corresponding to zero and non-zero eigenvalues are

$$
\left\{\left(\Delta U^{R}, V^{R}\right) \mid \Delta U^{R}=0\right\} \quad \text { and }\left\{\left(\Delta U^{R}, V^{R}\right) \mid V^{R}=0\right\}
$$

respectively. Using $\left(\Delta U^{R}, V^{R}\right)$, the system in $O_{\epsilon}^{i}$ becomes (5.23) and (5.24) (with $\lambda=0$ ) which captures the fast-slow behavior and exponential trichotomies of the dynamics. This new coordinates will be used in proving Lemma 5.3.

In inner layers, define

$$
V^{i}:=U_{\xi}^{i}-\left(D f-x_{0}^{i} I\right) U^{i}, \quad x \in I_{\epsilon}^{i} .
$$

The counter part of $\Delta U^{R}$ is undefined in inner layers. Therefore, for the coupled inner and outer system such as in Lemma 5.6, we will retain the variable $U$ together with the new variable $V$ as state variables.

We need to derive the jump conditions for $V^{R}$ and $V^{i}$, between outer and inner layers. Let $u_{\epsilon}$ be the exact Dafermos viscous shock solution. At each $x=x_{0}^{i}+\epsilon^{\beta}$, by (5.4), we have

$$
\begin{aligned}
& \epsilon U_{x}^{R}(x)-\left(D f\left(u_{\epsilon}(x)\right)-x I\right) U^{R}(x)-\left[U_{\xi}^{i}\left(\epsilon^{\beta-1}\right)-\left(D f\left(u_{\epsilon}(x)-x I\right) U^{i}\left(\epsilon^{\beta-1}\right)\right]\right. \\
= & \delta U_{\xi}^{i,+}-\left(D f\left(u_{\epsilon}(x)\right)-x I\right) \delta U^{i,+} .
\end{aligned}
$$


Replacing $D f\left(u_{\epsilon}\left(x_{0}^{i}+\epsilon^{\beta}\right)\right.$ by $D f\left(u_{0}^{R}\left(x_{0}^{i}+\epsilon^{\beta}\right)\right.$ in $O_{\epsilon}^{i}$, and by $D f\left(q^{i}\left(\epsilon^{\beta-1}\right)\right.$ in $I_{\epsilon}^{i}$, we have

$$
V^{R}\left(x_{0}^{i}+\epsilon^{\beta}\right)-V^{i}\left(\epsilon^{\beta-1}\right)=\delta U_{\xi}^{i,+}-\left(D f\left(u_{\epsilon}(x)\right)-x I\right) \delta U^{i,+}+N^{i,+}\left(U^{R}, U^{i}\right),
$$

where $x=x_{0}^{i}+\epsilon^{\beta}$, and the small term $N^{i,+}$ is

$$
N^{i,+}=\left[\left(D f\left(u_{0}^{R}\right)-D f\left(u_{\epsilon}\right)\right] U^{R}+\left[D f\left(u_{\epsilon}\right)-D f\left(q^{i}\right)\right] U^{i}=O\left(\epsilon\left(\left|U^{R}\right|+\left|U^{i}\right|\right)\right) .\right.
$$

Similarly,

$$
V^{i}\left(-\epsilon^{\beta-1}\right)-V^{R}\left(x_{0}^{i}-\epsilon^{\beta}\right)=\delta U_{\xi}^{i,-}-\left(D f\left(u_{\epsilon}(x)\right)-x I\right) \delta U^{i,-}+N^{i,-}\left(U^{R}, U^{i}\right),
$$

where $x=x_{0}^{i}-\epsilon^{\beta}$, and the small term $N^{i,-}$ is

$$
N^{i,-}=\left[D f\left(u_{\epsilon}\right)-\left(D f\left(u_{0}^{R}\right)\right] U^{R}+\left[D f\left(q^{i}\right)-D f\left(u_{\epsilon}\right)\right] U^{i}=O\left(\epsilon\left(\left|U^{R}\right|+\left|U^{i}\right|\right)\right) .\right.
$$

Dropping $N^{i, \pm}$ in (5.8) and (5.9), we consider the following jump conditions for the variables $V^{R}$ and $V^{i}$ :

$$
\begin{aligned}
V^{R}\left(x_{0}^{i}+\epsilon^{\beta}\right)-V^{i}\left(\epsilon^{\beta-1}\right) & =\delta V^{i,+}:=\delta U_{\xi}^{i,+}-\left(D f\left(u_{\epsilon}(x)\right)-x I\right) \delta U^{i,+}, \\
V^{i}\left(-\epsilon^{\beta-1}\right)-V^{R}\left(x_{0}^{i}-\epsilon^{\beta}\right) & =\delta V^{i,-}:=\delta U_{\xi}^{i,-}-\left(D f\left(u_{\epsilon}(x)\right)-x I\right) \delta U^{i,-} .
\end{aligned}
$$

For brevity, we denote $W=(U, V)$ and $\delta W^{i, \pm}=\left(\delta U^{i, \pm}, \delta V^{i, \pm}\right)$. See (5.4), and (5.10), (5.11) for these jumps.

Proposition 5.1. Consider the first order non-homogeneous systems (5.12)-(5.15) that is equivalent to (5.6) and (5.7). The coupled system in outer and inner layers is augmented by the jump conditions (5.16), (5.17) and boundary conditions (5.18):

$$
\begin{aligned}
& \epsilon U_{x}^{R}=(D f-x I) U^{R}+V^{R}, \\
& V_{x}^{R}=\left(\lambda_{0}+1\right) U^{R}+\lambda U_{1}^{R}+h^{R}(x), \\
& U_{\xi}^{i}=\left(D f-x_{0}^{i} I\right) U^{i}+V^{i}, \\
& V_{\xi}^{i}=\left[\left(\lambda_{0}+1\right) c^{i}+\lambda c_{0}^{i}\right] q_{\xi}^{i}+\left[\left(D^{2} f\left(q^{i}\right) u_{1}^{i}-\left(x_{1}^{i}+\xi\right) I\right) c^{i} q_{\xi}^{i}\right]_{\xi}+h^{i}(\xi), \\
& W^{R}\left(x_{0}^{i}+\epsilon^{\beta}\right)-W^{i}\left(\epsilon^{\beta-1}\right)=\delta W^{i,+}, \\
& W^{i}\left(-\epsilon^{\beta-1}\right)-W^{R}\left(x_{0}^{i}-\epsilon^{\beta}\right)=\delta W^{i,-}, \\
& \left(U^{R}, V^{R}\right)=O\left(e^{-\gamma|x|}\right), \quad \text { for } x \in R^{0}, R^{n} .
\end{aligned}
$$

The unknown parameters $\lambda$ and $\left\{c^{i}\right\}$ must also be solved from the system. Then there exists a unique solution $\left(U^{R}, V^{R},\left\{U^{i}\right\},\left\{V^{i}\right\}, \lambda,\left\{c^{i}\right\}\right)$ that satisfies the system. Moreover,

$$
\begin{aligned}
& \left|U^{R}\right|+\left|V^{R}\right|+\left|\left\{U^{i}\right\}\right|+\left|\left\{V^{i}\right\}\right|+\left|U^{R, 0}\right|_{\gamma}+\left|U^{R, n}\right|_{\gamma}+\left|V^{R, 0}\right|_{\gamma}+\left|V^{R, n}\right|_{\gamma}+|\lambda|+\left|\left\{c^{i}\right\}\right| \\
& \leq C\left(\left|\left\{\delta W^{i,+}\right\}\right|+\left|\left\{\delta W^{i,-}\right\}\right|+\left|h^{R}\right|+\epsilon^{\beta-1}\left|\left\{h^{i}\right\}\right|\right) .
\end{aligned}
$$

The proof of Proposition 5.1 depends on several lemmas and shall be deferred to the end of this section when all the lemmas are stated and proved. Among them, Lemma 5.3 treats nonhomogeneous systems without the concern of prescribed jumping conditions; Lemma 5.6 treats homogeneous systems with prescribed jumping conditions and boundary conditions at $R^{0}, R^{n}$. By adding results from the two lemmas, the entire system is solved by the super-position principle. 
Let $C(\gamma)$ be the space of continuous functions such that the norm

$$
\|U\|_{\gamma}=\sup _{x}\left\{|U(x)| e^{\gamma|x|}\right\}<\infty
$$

where the sup is taken over the domain $O_{\epsilon}^{0}$ or $O_{\epsilon}^{n}$. Let $L^{1}(\gamma)$ be the space of locally integrable functions such that the norm

$$
\|U\|_{L^{1}(\gamma)}=\int|U(x)| e^{\gamma|x|} d x<\infty
$$

where the integral is in the domain $O_{\epsilon}^{0}$ or $O_{\epsilon}^{n}$.

Lemma 5.2. (i) Let $T(x, y)$ be the principal matrix solution for the following equation in $O_{\epsilon}^{i}, 1 \leq i \leq n-1$ :

$$
\epsilon U_{x}-(D f-x I) U=h(x) .
$$

If $\epsilon>0$ is sufficiently small, then the system has exponential dichotomy on $O_{\epsilon}^{i}$ with super exponential decay rate, i.e., there exist projections $P_{u}(x)+P_{s}(x)=I$, exponent $\alpha / \epsilon>0$ and a constant $K$ that is independent of $\epsilon$, such that

$$
\begin{aligned}
& T(x, y) P_{s}(y)=P_{s}(x) T(x, y), \\
& \left|T(x, y) P_{s}(y)\right| \leq K e^{-\alpha(x-y) / \epsilon}, \quad x \geq y \\
& \left|T(x, y) P_{u}(y)\right| \leq K e^{-\alpha(y-x) / \epsilon}, \quad y \geq x .
\end{aligned}
$$

Moreover, in $O_{\epsilon}^{i}$, the rank of $P_{u}$ is $n-i$ and the rank of $P_{s}$ is $i$.

(ii) Consider the same equation (5.19) in $O_{\epsilon}^{0}$ or $O_{\epsilon}^{n}$. Then the solution is unstable in $O_{\epsilon}^{0}$ and stable in $O_{\epsilon}^{n}$ with super exponential decay rate:

$$
|T(x, y)| \leq K e^{-\alpha|x-y| / \epsilon}, \quad \text { for }\left\{\begin{array}{l}
x \leq y \in O_{\epsilon}^{0} \\
x \geq y \in O_{\epsilon}^{n}
\end{array}\right.
$$

(iii) For each $\lambda_{0} \in \mathbb{C}$, let $\Phi(x, y)$ be the principal matrix solution for the following equation in $O_{\epsilon}^{0}$ and $O_{\epsilon}^{n}$ :

$$
V_{x}+\left(\lambda_{0}+1\right)(D f-x I)^{-1} V=h(x) .
$$

Then the homogeneous part of the system has very slow growth rate either forward or backward in these layers. That is, for any $\delta>0$, there exists $K(\delta)>0$ such that

$$
|\Phi(x, y)| \leq K(\delta) e^{\delta|x-y|}, \quad x, y \in O_{\epsilon}^{0} \cup O_{\epsilon}^{n} .
$$

Moreover, for $h \in C(\gamma)$, or $h \in L^{1}(\gamma), \delta<\gamma$, there exists a unique solution $V \in C(\gamma)$. The following estimates hold

$$
|V|_{\gamma} \leq C|h|_{\gamma}, \quad|V|_{\gamma} \leq C|h|_{L^{1}(\gamma)}
$$

Proof. We prove Part (iii) only. There exist $\Theta>0$ such that for $|x| \geq \Theta$, we have $\left|\left(\lambda_{0}+1\right)(D f-x I)^{-1}\right|<\delta$. Thus, for $x, y \geq \Theta$ or $x, y<-\Theta$, we have $|\Phi(x, y)| \leq$ $K(\delta) e^{\delta|x-y|}$. The same estimates hold for $x, y \in O_{\epsilon}^{0} \cup O_{\epsilon}^{n}$ with maybe a larger $K(\delta)$, uniformly for $0<\epsilon<\epsilon_{0}$. For clarity, consider $O_{\epsilon}^{n}$. Let

$$
V(x)=\int_{\infty}^{x} \Phi(x, y) h(y) d y .
$$


Assuming that $\delta<\gamma$. Using the exponential estimate for $|\Phi(x, y)|$, it is elementary to verify that $V \in C(\gamma)$ if $h \in C(\gamma)$ or $L^{1}(\gamma)$ and the desired estimates hold.

We say that a solution of (5.19) satisfies the standard boundary condition on $O_{\epsilon}^{i}=$ $\left[a^{i}, b^{i}\right]$ if

$$
P_{s}\left(a^{i}\right) U\left(a^{i}\right)=0, \quad P_{u}\left(b^{i}\right) U\left(b^{i}\right)=0 .
$$

Lemma 5.3. Let $\lambda=0$. In outer layer, For each $h \in C\left(O_{\epsilon}^{i}\right), 1 \leq i \leq n-1$, system (5.12),(5.13) has a unique solution $\left(U^{R, i}, V^{R, i}\right)=\mathcal{F}^{R, i}\left(h^{R}\right)$ if $U^{R, i}$ satisfies the standard boundary condition on $O_{\epsilon}^{i}$ and $V^{R, i}\left(a^{i}\right)=0$. Moreover $\mathcal{F}^{R, i}$ is a bounded operator and the solutions satisfy

$$
\left|U^{R}\right|+\left|V^{R}\right| \leq C\left(\epsilon\left|h^{R}\right|+\left|h^{R}\right|_{L^{1}}\right), \quad\left|\Delta U^{R}\right| \leq C \epsilon\left(\left|h^{R}\right|+\left|h^{R}\right|_{L^{1}}\right) .
$$

In inner layers, assume $\lambda=0, c^{i}=0$. For each $h^{i} \in C\left(I_{\epsilon}^{i}\right)$, system (5.14),(5.15) has a unique solution $\left(U^{i}, V^{i}\right)=\mathcal{F}^{S, i}\left(h^{i}\right)$ that satisfies $U^{i}(0) \perp q^{i}(0), V^{i}(0)=0$. Moreover $\mathcal{F}^{S, i}$ is a bounded operator and the solution satisfies:

$$
\left|U^{i}\right|+\left|V^{i}\right| \leq C \epsilon^{\beta-1}\left|h^{i}\right| .
$$

Proof. The proof for system (5.14), (5.15) in inner layers shall be omitted.

In outer layers, if $\xi=x / \epsilon$ variable is used so that $\epsilon U_{x}=U_{\xi}$, then the length of the outer layer is $O(1 / \epsilon)$. If one uses variational of constant formula on (5.12) and (5.13) to get a solution with forcing term, then the desired estimates on the solution will not be satisfied. Exponential dichotomies must be used in the proof.

Consider the auxiliary equations

$$
\begin{aligned}
\epsilon U_{x}-(D f-x I) U & =h_{1}(x) \\
V_{x}+\left(\lambda_{0}+1\right)(D f-x I)^{-1} V & =h_{2}(x) .
\end{aligned}
$$

Using the standard boundary condition and exponential dichotomy on the first equation and the variation of constants formula on the second equation, we have the unique solutions

$$
\begin{array}{cc}
U=\mathcal{F}_{1}\left(h_{1}\right), & V=\mathcal{F}_{2}\left(h_{2}\right), \\
\mathcal{F}_{1}: C\left(O_{\epsilon}^{i}\right) \rightarrow C\left(O_{\epsilon}^{i}\right), & \mathcal{F}_{2}: L^{1}\left(O_{\epsilon}^{i}\right) \rightarrow C\left(O_{\epsilon}^{i}\right), \\
|U| \leq C\left|h_{1}\right|, & \left|V^{R}\right| \leq C\left|h_{2}\right|_{L^{1}} .
\end{array}
$$

Using $\left(\Delta U^{R}, V^{R}\right),(5.12),(5.13)$, with $\lambda=0$, become

$$
\begin{aligned}
& \epsilon \Delta U_{x}^{R}-(D f-x I) \Delta U^{R}=\epsilon\left[G\left(\Delta U^{R}, V^{R}\right)+(D f-x I)^{-1} h^{R}\right], \\
& V_{x}^{R}+\left(\lambda_{0}+1\right)(D f-x I)^{-1} V^{R}=\left(\lambda_{0}+1\right) \Delta U^{R}+h^{R} .
\end{aligned}
$$

Here

$$
\begin{aligned}
G\left(\Delta U^{R}, V^{R}\right)=\left(\lambda_{0}+1\right) & (D f-x I)^{-1} \Delta U^{R} \\
+ & {\left[(D f-x I)_{x}^{-1}-\left(\lambda_{0}+1\right)(D f-x I)^{-1}(D f-x I)^{-1}\right] V^{R} . }
\end{aligned}
$$


System (5.23), (5.24) can be written as a fixed point problem

$$
\begin{aligned}
\Delta U_{1}^{R} & =\epsilon \mathcal{F}_{1}\left(G\left(\Delta U^{R}, V^{R}\right)+(D f-x I)^{-1} h^{R}\right), \\
V_{1}^{R} & =\mathcal{F}_{2}\left(\left(\lambda_{0}+1\right) \Delta U_{1}^{R}+h^{R}\right) .
\end{aligned}
$$

The mapping defined by $\left(\Delta U^{R}, V^{R}\right) \rightarrow\left(\Delta U_{1}^{R}, V_{1}^{R}\right)$ is a contraction if $\epsilon$ is sufficiently small. Therefore, there is a unique fixed point $\left(\Delta U^{R}, V^{R}\right)$ which is also a solution to the coupled system $(5.23),(5.24)$.

Since $\mathcal{F}_{1}$ is a bounded operator, we have $\left|\Delta U^{R}\right| \leq C \epsilon\left(\left|\Delta U^{R}\right|+\left|V^{R}\right|+\left|h^{R}\right|\right)$. This simplifies to

$$
\left|\Delta U^{R}\right| \leq C \epsilon\left(\left|V^{R}\right|+\left|h^{R}\right|\right)
$$

On the other hand since $\mathcal{F}_{2}$ is a bounded operator,

$$
\left|V^{R}\right| \leq C_{1}\left(\left|\Delta U^{R}\right|+\left|h^{R}\right|_{L^{1}}\right) \leq C_{1}\left(C \epsilon\left(\left|V^{R}\right|+\left|h^{R}\right|\right)+\left|h^{R}\right|_{L^{1}}\right) .
$$

Thus $\left|V^{R}\right| \leq C\left(\epsilon\left|h^{R}\right|+\left|h^{R}\right|_{L^{1}}\right)$. Plug back into the estimate for $\left|\Delta U^{R}\right|$, we have $\left|\Delta U^{R}\right| \leq C \epsilon\left(\left|h^{R}\right|+\left|h^{R}\right|_{L^{1}}\right)$.

Finally $\left|U^{R}\right| \leq C\left(\left|\Delta U^{R}\right|+\left|V^{R}\right|\right) \leq C\left(\epsilon\left|h^{R}\right|+\left|h^{R}\right|_{L^{1}}\right)$.

Lemma 5.4. (i) In the semi-infinite outer layers $R^{0}$ and $R^{n}$, let $h_{1} \in C(\gamma)$ and $\phi \in \mathbb{R}^{n}$. Then There exists a unique solutions $U \in C(\gamma)$ for the boundary value problem

$$
\epsilon U_{x}=(D f-x I) U+h_{1},\left\{\begin{array}{l}
U\left(x_{0}^{1}\right)=\phi_{u}^{R, 0}, x \in R^{0}, \\
U\left(x_{0}^{n}\right)=\phi_{s}^{R, n}, x \in R^{n} .
\end{array}\right.
$$

(ii) Let $h_{2} \in C(\gamma)$ or $L^{1}(\gamma)$. Then there exists a unique solutions $V \in C(\gamma)$ for the single equation without boundary conditions

$$
V_{x}+\left(\lambda_{0}+1\right)(D f-x I)^{-1} V=h_{2} .
$$

(iii) For $h^{R} \in C(\gamma) \cap L^{1}(\gamma)$, consider the non-homogeneous boundary value problem

$$
\begin{aligned}
& \epsilon U_{x}=(D f-x I) U+V, \quad\left\{\begin{array}{l}
U\left(x_{0}^{1}\right)=0, x \in R^{0}, \\
U\left(x_{0}^{n}\right)=0, x \in R^{n} .
\end{array}\right. \\
& V_{x}=\left(\lambda_{0}+1\right) U^{R}+h^{R},
\end{aligned}
$$

Then, there exists a unique solution $(U, V) \in C(\gamma)$ such that

$$
|U|_{\gamma}+|V|_{\gamma} \leq C\left(\epsilon\left|h^{R}\right|_{\gamma}+\left|h^{R}\right|_{L^{1}(\gamma)}\right)
$$

Moreover, let $\Delta U^{R}=U+(D f-x I)^{-1} V$, then

$$
\left|\Delta U^{R}\right|_{\gamma} \leq C \epsilon\left(\left|h^{R}\right|_{\gamma}+\left|h^{R}\right|_{L^{1}(\gamma)}\right) .
$$

Proof. We shall only prove the case $x \in O_{\epsilon}^{0}$ only. The proof is a mimic of that of Lemma 5.3.

For brevity, let $a^{0}=-\infty, b^{0}=x_{0}^{1}-\epsilon^{\beta}, O_{\epsilon}^{0}=\left(-\infty, b^{0}\right)$.

Proof of Part (i): The unique solution $U \in C(\gamma)$ of (5.25) can be written as

$$
U^{R, i}(x)=\int_{b^{0}}^{x} T(x, y) P_{u}(y) h_{1}(y) d y+T\left(x, b^{0}\right) \phi_{u}^{R, 0} .
$$


Using exponential dichotomy on $O_{\epsilon}^{0}$, we have

$$
U=\mathcal{F}_{1}\left(h_{1}, \phi_{u}^{R, 0}\right), \quad|U|_{\gamma} \leq C\left(\left|h_{1}\right|_{\gamma}+\left|\phi_{u}^{R, 0}\right|\right) .
$$

Proof of Part (ii): The unique solution $V \in C(\gamma)$ of (5.26) can be written as

$$
V^{R, i}(x)=\int_{-\infty}^{x} \Phi(x, y) h_{2}(y) d y
$$

Using exponential estimate in $O_{\epsilon}^{0}$ we have

$$
\left|V^{R}\right|_{\gamma}=\mathcal{F}_{2}\left(h_{2}\right), \quad\left|V^{R}\right| \leq C\left|h_{2}\right|_{\gamma} \text { or } C\left|h^{2}\right|_{L^{1}(\gamma)} .
$$

Proof of Part (iii): Using $\left(\Delta U^{R}, V^{R}\right),(5.27),(5.28)$, become

$$
\begin{aligned}
& \epsilon \Delta U_{x}^{R}-(D f-x I) \Delta U^{R}=\epsilon\left[G\left(\Delta U^{R}, V^{R}\right)+(D f-x I)^{-1} h^{R}\right], \\
& V_{x}^{R}+\left(\lambda_{0}+1\right)(D f-x I)^{-1} V^{R}=\left(\lambda_{0}+1\right) \Delta U^{R}+h^{R} .
\end{aligned}
$$

Here

$$
\begin{aligned}
G\left(\Delta U^{R}, V^{R}\right)=\left(\lambda_{0}+1\right) & (D f-x I)^{-1} \Delta U^{R} \\
+ & {\left[(D f-x I)_{x}^{-1}-\left(\lambda_{0}+1\right)(D f-x I)^{-1}(D f-x I)^{-1}\right] V^{R} . }
\end{aligned}
$$

System (5.29), (5.30) can be written as a fixed point problem

$$
\begin{aligned}
\Delta U_{1}^{R} & =\epsilon \mathcal{F}_{1}\left(G\left(\Delta U^{R}, V^{R}\right)+(D f-x I)^{-1} h^{R}, 0\right), \\
V_{1}^{R} & =\mathcal{F}_{2}\left(\left(\lambda_{0}+1\right) \Delta U_{1}^{R}+h^{R}\right) .
\end{aligned}
$$

The mapping defined by $\left(\Delta U^{R}, V^{R}\right) \rightarrow\left(\Delta U_{1}^{R}, V_{1}^{R}\right)$ is a contraction if $\epsilon$ is sufficiently small. Therefore, there is a unique fixed point $\left(\Delta U^{R}, V^{R}\right)$ which is also a solution to the coupled system (5.23),(5.24).

Since $\mathcal{F}_{1}$ is a bounded operator, we have $\left|\Delta U^{R}\right|_{\gamma} \leq C \epsilon\left(\left|\Delta U^{R}\right|_{\gamma}+\left|V^{R}\right|_{\gamma}+\left|h^{R}\right|_{\gamma}\right)$. This simplifies to

$$
\left|\Delta U^{R}\right|_{\gamma} \leq C \epsilon\left(\left|V^{R}\right|_{\gamma}+\left|h^{R}\right|_{\gamma}\right)
$$

On the other hand since $\mathcal{F}_{2}$ is a bounded operator,

$$
\left|V^{R}\right|_{\gamma} \leq C_{1}\left(\left|\Delta U^{R}\right|_{\gamma}+\left|h^{R}\right|_{L^{1}}\right) \leq C_{1}\left(C \epsilon\left(\left|V^{R}\right|_{\gamma}+\left|h^{R}\right|_{\gamma}\right)+\left|h^{R}\right|_{L^{1}(\gamma)}\right)
$$

Thus $\left|V^{R}\right|_{\gamma} \leq C\left(\epsilon\left|h^{R}\right|_{\gamma}+\left|h^{R}\right|_{L^{1}(\gamma)}\right)$. Plug back into the estimate for $\left|\Delta U^{R}\right|$, we have $\left|\Delta U^{R}\right|_{\gamma} \leq C \epsilon\left(\left|h^{R}\right|_{\gamma}+\left|h^{R}\right|_{L^{1}(\gamma)}\right)$.

Finally $\left|U^{R}\right|_{\gamma} \leq C\left(\left|\Delta U^{R}\right|_{\gamma}+\left|V^{R}\right|_{\gamma}\right) \leq C\left(\epsilon\left|h^{R}\right|_{\gamma}+\left|h^{R}\right|_{L^{1}(\gamma)}\right)$.

Lemma 5.5. Consider the system of $V$-equations in $O_{\epsilon}^{i}, 0 \leq i \leq n$ and $I_{\epsilon}^{i}, 1 \leq i \leq n$, with $h^{R}=0$ and $h^{i}=0$ :

$$
\begin{aligned}
& V_{x}^{R}+\left(\lambda_{0}+1\right)(D f-x I)^{-1} V^{R}=\lambda U_{1}^{R}, \\
& V_{\xi}^{i}=\left[\left(\lambda_{0}+1\right) c^{i}+\lambda c_{0}^{i}\right] q_{\xi}^{i}+\left[\left(D^{2} f\left(q^{i}\right) u_{1}^{i}-\left(x_{1}^{i}+\xi\right) I\right) c^{i} q_{\xi}^{i}\right]_{\xi}, \\
& V^{R}\left(x_{0}^{i}+\epsilon^{\beta}\right)-V^{i}\left(\epsilon^{\beta-1}\right)=\delta V^{i,+} \\
& V^{i}\left(-\epsilon^{\beta-1}\right)-V^{R}\left(x_{0}^{i}-\epsilon^{\beta}\right)=\delta V^{i,-}, \\
& V^{R}=O\left(e^{-\gamma|x|}\right), \quad \text { for } x \in R^{0}, R^{n} .
\end{aligned}
$$


Then the above system has a unique solution $\left(V^{R},\left\{V^{i}\right\},\left\{c^{i}\right\}, \lambda\right)$. Moreover,

$$
\left|V^{R}\right|+\left|\left\{V^{i}\right\}\right|+\left|\left\{c^{i}\right\}\right|+|\lambda| \leq C\left(\left|\left\{\delta V^{i,-}\right\}\right|+\left|\left\{\delta V^{i,+}\right\}\right|\right) .
$$

Proof. Let $\Delta_{\epsilon}^{i}:=q^{i}\left(\epsilon^{\beta-1}\right)-q^{i}\left(-\epsilon^{\beta-1}\right)$. The second equation in $I_{\epsilon}^{i}$ can be integrated once to yield

$$
V^{i}\left(\epsilon^{\beta-1}\right)-V^{i}\left(-\epsilon^{\beta-1}\right)=\left[\left(\lambda_{0}+1\right) c^{i}+\lambda c_{0}^{i}\right] \Delta_{\epsilon}^{i}+O\left(e^{-\alpha \epsilon^{\beta-1}}\left|c^{i}\right|\right) .
$$

The small factor $e^{-\alpha \epsilon^{\beta-1}}$ comes from $q_{\xi}^{i}(\xi)$ for $\xi=\epsilon^{\beta-1}$. The small term can be handled by contraction mapping principle and shall be omitted for convenience. Consider

$$
V^{i}\left(\epsilon^{\beta-1}\right)-V^{i}\left(-\epsilon^{\beta-1}\right)=\left[\left(\lambda_{0}+1\right) c^{i}+\lambda c_{0}^{i}\right] \Delta_{\epsilon}^{i} .
$$

Combining this with $\delta V^{i,-}$ and $\delta V^{i,-}$, we find that

$$
V^{R}\left(x_{0}^{i}+\epsilon^{\beta}\right)-V^{R}\left(x_{0}^{i}-\epsilon^{\beta}\right)=\left[\left(\lambda_{0}+1\right) c^{i}+\lambda c_{0}^{i}\right] \Delta_{\epsilon}^{i}+\delta Z^{i},
$$

where

$$
\delta Z^{i}:=\delta V^{i,-}+\delta V^{i,+} .
$$

In the spirit of SLEP method, the whole system reduces to a system in outer layers coupled with jump conditions:

$$
\begin{aligned}
& V_{x}^{R}+\left(\lambda_{0}+1\right)(D f-x I)^{-1} V^{R}=\lambda U_{1}^{R}, \\
& V^{R}\left(x_{0}^{i}+\epsilon^{\beta}\right)-V^{R}\left(x_{0}^{i}-\epsilon^{\beta}\right)=\left[\left(\lambda_{0}+1\right) c^{i}+\lambda c_{0}^{i}\right] \Delta_{\epsilon}^{i}+\delta Z^{i} .
\end{aligned}
$$

Let

$$
Z^{R}:=(D f-x I)^{-1} V^{R}
$$

We have $V^{R}=(D f-x I) Z^{R}$. The system in outer layers can be written as

$$
\begin{aligned}
& \left((D f-x I) Z^{R}\right)_{x}+\left(\lambda_{0}+1\right) Z^{R}=\lambda U_{1}^{R}, \\
& (D f-x I) Z^{R}\left(x_{0}^{i}+\epsilon^{\beta}\right)-(D f-x I) Z^{R}\left(x_{0}^{i}-\epsilon^{\beta}\right) \\
& =\left[\left(\lambda_{0}+1\right) c^{i}+\lambda c_{0}^{i}\right] \Delta_{\epsilon}^{i}+\delta Z^{i} .
\end{aligned}
$$

If we substitute $\Delta_{\epsilon}^{i}$ by $\Delta^{i}+q^{i}\left(\epsilon^{\beta-1}\right)-q^{i}(\infty)+q^{i}(-\infty)-q^{i}\left(-\epsilon^{\beta-1}\right)$ and add the following equations to $(5.33)$

$$
\begin{aligned}
& (D f-x I) Z^{i}\left(x_{0}^{i}+\right)-(D f-x I) Z^{i}\left(x_{0}^{i}+\epsilon^{\beta}\right) \\
= & -\int_{x_{0}^{i}}^{x_{0}^{i}+\epsilon^{\beta}}\left[\lambda U_{1}^{R}+h^{R}-\left(\lambda_{0}+1\right) Z^{R}(y)\right] d y, \\
& (D f-x I) Z^{i}\left(x_{0}^{i}-\epsilon^{\beta}\right)-(D f-x I) Z^{i}\left(x_{0}^{i}-\right) \\
= & -\int_{x_{0}^{i}-\epsilon^{\beta}}^{x_{0}^{i}-}\left[\lambda U_{1}^{R}+h^{R}-\left(\lambda_{0}+1\right) Z^{R}(y)\right] d y,
\end{aligned}
$$

we have

$(D f-x I) Z^{i}\left(x_{0}^{i}+\right)-(D f-x I) Z^{i}\left(x_{0}^{i}-\right)=\left[\left(\lambda_{0}+1\right) c^{i}+\lambda c_{0}^{i}\right] \Delta^{i}+\delta Z^{i}+N\left(\lambda, c^{i}, Z^{R}\right)$, 
where

$$
\begin{gathered}
N\left(\lambda, c^{i}, Z^{R}\right)=\left[\left(\lambda_{0}+1\right) c^{i}+\lambda c_{0}^{i}\right]\left(q^{i}\left(\epsilon^{\beta-1}\right)-q^{i}(\infty)+q^{i}(-\infty)-q^{i}\left(-\epsilon^{\beta-1}\right)\right. \\
\quad-\int_{x_{0}^{i}-\epsilon^{\beta}}^{x_{0}^{i}-}\left[\lambda U_{1}^{R}-\left(\lambda_{0}+1\right) Z^{R}(y)\right] d y-\int_{x_{0}^{i}}^{x_{0}^{i}+\epsilon^{\beta}}\left[\lambda U_{1}^{R}-\left(\lambda_{0}+1\right) Z^{R}(y)\right] d y
\end{gathered}
$$

is a small term.

Consider a simplified problem

$$
\begin{aligned}
& \left((D f-x I) Z^{R}\right)_{x}+\left(\lambda_{0}+1\right) Z^{R}=\lambda U_{1}^{R}, \\
& (D f-x I) Z^{i}\left(x_{0}^{i}+\right)-(D f-x I) Z^{i}\left(x_{0}^{i}-\right)=\left[\left(\lambda_{0}+1\right) c^{i}+\lambda c_{0}^{i}\right] \Delta^{i}+\delta Z^{i} .
\end{aligned}
$$

Note that from Lemma 5.4, $Z^{0}$ and $Z^{n}$ can be solved first without the jump conditions. Then to solve for $Z^{i}, 1 \leq i \leq n-1$, replacing $Z^{0}, Z^{n}$ by zeros, and consider the abstract system

$$
\begin{aligned}
\mathcal{L}\left(\left\{Z^{R i}\right\}_{0}^{n},\left\{c^{i} \Delta^{i}\right\}_{1}^{n}\right) & +\left(\lambda_{0}+1\right)\left(\left\{Z^{R i}\right\}_{0}^{n},\left\{c^{i} \Delta^{i}\right\}_{1}^{n}\right) \\
& +\lambda\left(\left\{U_{1}^{R i}\right\}_{0}^{n},\left\{c_{0}^{i} \Delta^{i}\right\}_{1}^{n}\right)=\left(\left\{W^{i}\right\}_{0}^{n},\left\{J^{i}\right\}_{1}^{n}\right),
\end{aligned}
$$

where

$$
\begin{aligned}
& J^{i}=\delta Z^{i}, \quad 2 \leq i \leq n-1, \\
& J^{1}=\delta Z^{1}+Z^{R}\left(x_{0}^{1}-\epsilon^{\beta}\right), \\
& J^{n}=\delta Z^{n}-Z^{R}\left(x_{0}^{n}+\epsilon^{\beta}\right) .
\end{aligned}
$$

By the result of section 4 , the above system has a unique solution, denoted by

$$
\left(Z^{R}, \lambda,\left\{c^{i}\right\}\right)=\mathcal{F}\left(\left\{\delta Z^{i}\right\}\right)
$$

Then the original system can be written as a fixed point problem:

$$
\left(Z^{R}, \lambda,\left\{c^{i}\right\}\right)=\mathcal{F}\left(\left\{\delta Z^{i}\right\}+N\left(\lambda,\left\{c^{i}\right\}, Z^{R}\right)\right) .
$$

By contraction mapping principle, the above has a unique solution.

Lemma 5.6. Consider the linear homogeneous system

$$
\begin{aligned}
& \epsilon U_{x}^{R}=(D f-x I) U^{R}+V^{R}, \\
& V_{x}^{R}=\left(\lambda_{0}+1\right) U^{R}+\lambda U_{1}^{R} . \\
& U_{\xi}^{i}=\left(D f-x_{0}^{i} I\right) U^{i}+V^{i}, \\
& V_{\xi}^{i}=\left[\left(\lambda_{0}+1\right) c^{i}+\lambda c_{0}^{i}\right] q_{\xi}^{i}+\left[\left(D^{2} f\left(q^{i}\right) u_{1}^{i}-\left(x_{1}^{i}+\xi\right) I\right) c^{i} q_{\xi}^{i}\right]_{\xi} .
\end{aligned}
$$

with jump conditions and boundary conditions:

$$
\begin{aligned}
& W^{R}\left(x_{0}^{i}+\epsilon^{\beta}\right)-W^{i}\left(\epsilon^{\beta-1}\right)=\delta W^{i,+}, \\
& W^{i}\left(-\epsilon^{\beta-1}\right)-W^{R}\left(x_{0}^{i}-\epsilon^{\beta}\right)=\delta W^{i,-}, \\
& \left(U^{R}, V^{R}\right)=O\left(e^{-\gamma|x|}\right), \quad \text { for } x \in R^{0}, R^{n} .
\end{aligned}
$$


Then there exists a unique solution to the homogeneous system with the prescribed boundary condition and jump conditions. Moreover,

$$
\begin{aligned}
& \left|U^{R}\right|+\left|V^{R}\right|+\left|\left\{U^{i}\right\}\right|+\left|\left\{V^{i}\right\}\right|+\left|U^{R, 0}\right|_{\gamma}+\left|U^{R, n}\right|_{\gamma}+\left|V^{R, 0}\right|_{\gamma}+\left|V^{R, n}\right|_{\gamma} \\
& \leq C\left(\left|\left\{\delta W^{i,-}\right\}\right|+\left|\left\{\delta W^{i,+}\right\}\right|\right) .
\end{aligned}
$$

Proof. As before, let $\Delta U^{R}=U^{R}+(D f-x I)^{-1} V^{R}$. The second equation (5.35) of the system is equivalent to

$$
V_{x}^{R}+\left(\lambda_{0}+1\right)(D f-x I)^{-1} V^{R}=\left(\lambda_{0}+1\right) \Delta U^{R}+\lambda U_{1}^{R}
$$

The proof is divided into three steps:

STEP 1: Dropping $\left(\lambda_{0}+1\right) \Delta U^{R}$ in (5.41), we have a simplified equation for $V^{R}$. This is coupled with (5.37) to be a system of $V$-equations, and will be solved with jump and boundary conditions (5.38)-(5.40). We will determine approximate values of $\lambda$ and $c^{i}, 1 \leq i \leq n$ at this stage also. The result is stated in Lemma 5.5.

STEP 2: With $\left(V^{R}, V^{i}\right)$ obtained from STEP 1, we solve a coupled system of $U$-equations with jump and boundary conditions:

$$
\begin{aligned}
& \epsilon U_{x}^{R}=(D f-x I) U^{R}+V^{R}, \\
& U_{\xi}^{i}=\left(D f-x_{0}^{i} I\right) U^{i}+V^{i}, \\
& U^{R}\left(x_{0}^{i}+\epsilon^{\beta}\right)-U^{i}\left(\epsilon^{\beta-1}\right)=\delta U^{i,+}, \\
& U^{i}\left(-\epsilon^{\beta-1}\right)-U^{R}\left(x_{0}^{i}-\epsilon^{\beta}\right)=\delta U^{i,-}, \\
& U^{R}=O\left(e^{-\gamma|x|}\right), \quad \text { for } x \in R^{0}, R^{n} .
\end{aligned}
$$

For brevity, let $a^{i}=x_{0}^{i}+\epsilon^{\beta}, b^{i}=x_{0}^{i+1}-\epsilon^{\beta}$. Then $O_{\epsilon}^{i}=\left\{a^{i} \leq x \leq b^{i}\right\}$. See Figure 5.1 for the illustration of symbols used in this proof.

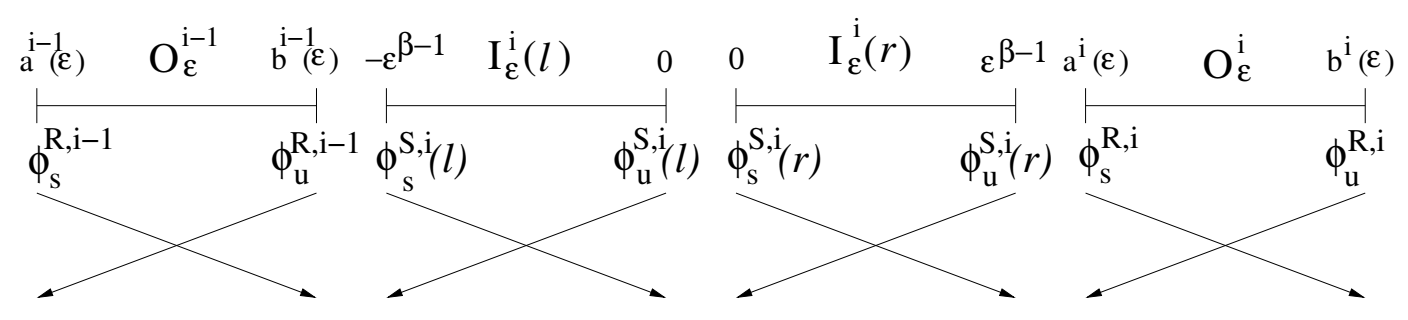

Figure 5.1. Notations for the initial data $\phi_{u}^{R, i}$, etc. The solutions passing through them decay exponentially in the direction indicated by the arrows.

In $O_{\epsilon}^{i}$ using exponential dichotomies, we have

$$
\begin{aligned}
U^{R, i}(x) & =\int_{a^{i}}^{x} T(x, y) P_{s}(y) V^{R, i}(y) d y+\int_{b^{i}}^{x} T(x, y) P_{u}(y) V^{R, i}(y) d y \\
& +T\left(x, a^{i}\right) \phi_{s}^{R, i}+T\left(x, b^{i}\right) \phi_{u}^{R, i} .
\end{aligned}
$$

Let $I_{\epsilon}^{i}=I_{\epsilon}^{i}(\ell) \cup I_{\epsilon}^{i}(r)$ where

$$
I_{\epsilon}^{i}(\ell)=\left(-\epsilon^{\beta-1}, 0\right), \quad I_{\epsilon}^{i}(r)=\left(0, \epsilon^{\beta-1}\right) .
$$


In each of the subintervals $I_{\epsilon}^{i}(\ell)$ and $I_{\epsilon}^{i}(r)$, using exponential dichotomies, we have

$$
\begin{aligned}
U^{i}(\xi) & =\int_{-\epsilon^{\beta-1}}^{\xi} S(\xi, \eta) P_{s}(\eta) V^{i}(\eta) d \eta+\int_{0}^{\xi} S(\xi, \eta) P_{u}(\eta) V^{i}(\eta) d \eta \\
& +S\left(\xi,-\epsilon^{\beta-1}\right) \phi_{s}^{S, i}(\ell)+S(\xi, 0) \phi_{u}^{S, i}(\ell) \quad \xi \in I_{\epsilon}^{i}(\ell), \\
U^{i}(\xi) & =\int_{0}^{\xi} S(\xi, \eta) P_{s}(\eta) V^{i}(\eta) d \eta+\int_{\epsilon^{\beta-1}}^{\xi} S(\xi, \eta) P_{u}(\eta) V^{i}(\eta) d \eta \\
& +S\left(\xi, \epsilon^{\beta-1}\right) \phi_{u}^{S, i}(r)+S(\xi, 0) \phi_{u}^{S, i}(r) \quad \xi \in I_{\epsilon}^{i}(r) .
\end{aligned}
$$

To satisfy the jump conditions, we need

$$
\begin{aligned}
\phi_{s}^{R, i}-\phi_{u}^{S, i}(r) & =\int_{0}^{\epsilon^{\beta-1}} S\left(\epsilon^{\beta-1}, \eta\right) P_{s}(\eta) V^{i}(\eta) d \eta-\int_{b^{i}}^{a^{i}} T\left(a^{i}, y\right) P_{u}(y) V^{R, i} d y \\
& +S\left(\epsilon^{\beta-1}, 0\right) \phi_{s}^{S, i}(r)-T\left(a^{i}, b^{i}\right) \phi_{u}^{R, i}+\delta U^{i,+} \\
\phi_{s}^{S, i}(\ell)-\phi_{u}^{R, i-1} & =\int_{a^{i-1}}^{b^{i-1}} P_{s}(y) V^{R, i-1}(y) d y-\int_{0}^{-\epsilon^{\beta-1}} S\left(-\epsilon^{\beta-1}, \eta\right) P_{u}(\eta) V^{i}(\eta) d \eta \\
& +T\left(b^{i-1}, a^{i-1}\right) \phi_{s}^{R, i-1}-S\left(-\epsilon^{\beta-1}, 0\right) \phi_{u}^{S, i}(\ell)+\delta U^{i,-} .
\end{aligned}
$$

In the right hand sides, if $\left(\phi_{s}^{S, i}(r), \phi_{u}^{R, i}\right)$ of the first equation and $\left(\phi_{s}^{R, i-1}, \phi_{u}^{S, i}(\ell)\right)$ of the second equation are given, then using transverse intersections of stable and unstable subspaces, we can calculate terms in the left hand sides: $\left(\phi_{s}^{R, i}, \phi_{u}^{S, i}(r)\right)$ of the first equation, and $\left(\phi_{s}^{S, i}(\ell), \phi_{u}^{R, i-1}\right)$ of the second equation. Then from the lemmas, we have $\left(U^{R, i}, U^{S, i}\right)$, from which the terms in the right hand sides: $\phi_{s}^{S, i}(r), \phi_{u}^{R, i}, \phi_{s}^{R, i-1}, \phi_{u}^{S, i}(\ell)$ can be calculated again. The above can be viewed as an fixed point problem:

$$
\begin{aligned}
\left(\phi_{s}^{R, i}, \phi_{u}^{R, i}, \phi_{u}^{S, i}(\ell), \phi_{s}^{S, i}(r)\right) & \rightarrow\left(\phi_{s}^{R, i}, \phi_{u}^{R, i}, \phi_{s}^{S, i}(\ell), \phi_{u}^{S, i}(r)\right) \\
& \rightarrow\left(U^{R, i}, U^{S, i}\right) \\
& \rightarrow\left(\phi_{s}^{R, i}, \phi_{u}^{R, i}, \phi_{u}^{S, i}(\ell), \phi_{s}^{S, i}(r)\right)
\end{aligned}
$$

Owing to the exponential decay of

$$
\left.S\left(\epsilon^{\beta-1}, 0\right) \phi_{s}^{S, i}(r), \quad T\left(b^{i-1}, a^{i-1}\right) \phi_{s}^{R, i-1}, \quad T\left(a^{i}\right), b^{i}\right) \phi_{u}^{R, i}, \quad S\left(-\epsilon^{\beta-1}, 0\right) \phi_{u}^{S, i}(\ell),
$$

the process in (5.42) is also a contraction mapping. Therefore it has a unique fixed point $\left(\phi_{s}^{R, i}, \phi_{u}^{R, i}, \phi_{u}^{S, i}(\ell), \phi_{s}^{S, i}(r)\right)$, which can be used to determine $\left(U^{R, i}, U^{S, i}\right)$.

With $\left(U^{R}, U^{i}, V^{R}, V^{i}, \lambda, c^{i}\right)$ from STEP1 and STEP 2, (5.41) is satisfied without the term $\left(\lambda_{0}+1\right) \Delta U^{R}$. Now using this $U^{R}$ and $V^{R}$, define $\Delta U^{R}=U^{R}+\left(D f\left(u_{0}^{R}\right)-\right.$ $x I)^{-1} V^{R}$. We show that $\Delta U^{R}$ is small in suitable norms. We can verify that

$$
\Delta U_{\xi}^{R}-(D f-x I) \Delta U^{R}=\epsilon G\left(V^{R}, \lambda\right) .
$$

Where

$$
G\left(V^{R}, \lambda\right)=\left[(D f-x I)_{x}^{-1}-\left(\lambda_{0}+1\right)(D f-x I)^{-1}(D f-x I)^{-1}\right] V^{R}+\lambda(D f-x I)^{-1} U_{1}^{R} .
$$


Using the exponential dichotomy in $O_{\epsilon}^{i}$, we have

$$
\begin{aligned}
\Delta U^{R}(\xi) & =\int_{a^{i}}^{x} T(x, y) P_{s}(y) \epsilon\left[G\left(V^{R}, \lambda\right)\right](y) d y \\
& +\int_{b^{i}}^{x} T(x, y) P_{u}(y) \epsilon\left[G\left(V^{R}, \lambda\right)\right] d y \\
& +T\left(x, a^{i}\right) P_{s}\left(a^{i}\right) \Delta U^{R}\left(a^{i}\right)+T\left(x, b^{i}\right) P_{u}\left(b^{i}\right) \Delta U^{R}\left(b^{i}\right) .
\end{aligned}
$$

By definition,

$$
\left|\Delta U^{R}\right| \leq\left|U^{R}\right|+\left|(D f-x I)^{-1}\right|\left|V^{R}\right| \leq C\left(\left|\left\{\delta W^{i,-}\right\}\right|+\left|\left\{\delta W^{i,+}\right\}\right|\right) .
$$

If we observe that

$$
\left|G\left(V^{R}, \lambda\right)\right| \leq C\left(\left|V^{R}\right|+|\lambda|\right) \leq C\left(\left|\left\{\delta W^{i,-}\right\}\right|+\left|\left\{\delta W^{i,+}\right\}\right|\right),
$$

then the sup norms of the two integrals of (5.43) are bounded by $C \epsilon\left(\left|\delta W^{i, \pm}\right|\right)$. Therefore, their $L^{1}(x)$ norms are bounded by $C \epsilon\left(\left|\delta W^{i, \pm}\right|\right)$. Due to the large negative exponential rate $-\alpha / \epsilon$, the two boundary terms,

$$
T\left(x, a^{i}\right) P_{s}\left(a^{i}\right) \Delta U^{R}\left(a^{i}\right)+T\left(x, b^{i}\right) P_{u}\left(b^{i}\right) \Delta U^{R}\left(b^{i}\right),
$$

are bounded by $\epsilon\left|\Delta U^{R}\right| \leq C \epsilon\left(\left|\delta W^{i, \pm}\right|\right)$ in $L^{1}(x)$ norm.

In summary

$$
\left|\Delta U^{R}\right|_{\infty} \leq C\left(\left|\left\{\delta W^{i,-}\right\}\right|+\left|\left\{\delta W^{i,+}\right\}\right|\right), \quad\left|\Delta U^{R}\right|_{L^{1}(x)} \leq C \epsilon\left(\left|\left\{\delta W^{i,-}\right\}\right|+\left|\left\{\delta W^{i,+}\right\}\right|\right) .
$$

STEP 3: Consider the following system in $O_{\epsilon}^{i}$ :

$$
\begin{aligned}
& \epsilon \bar{U}_{x}^{R}=(D f-x I) \bar{U}^{R}+\bar{V}^{R}, \\
& \bar{V}_{x}^{R}+\left(\lambda_{0}+1\right)(D f-x I)^{-1} \bar{V}^{R}=\left(\lambda_{0}+1\right) \Delta \bar{U}^{R}+\left(\lambda_{0}+1\right) \Delta U^{R} .
\end{aligned}
$$

Using Lemma 5.3 with $\left(\lambda_{0}+1\right) \Delta U^{R}$ as a forcing term, the above system has a solution that satisfies

$$
\left|\bar{U}^{R}\right|+\left|\bar{V}^{R}\right| \leq C\left(\epsilon\left|\Delta U^{R}\right|+\left|\Delta U^{R}\right|_{L^{1}}\right) \leq C \epsilon\left(\left|\left\{\delta W^{i,-}\right\}\right|+\left|\left\{\delta W^{i,+}\right\}\right|\right) .
$$

By adding $\left(\bar{U}^{R}, \bar{V}^{R}\right)$ to $\left(U^{R}, V^{R}\right)$ obtained in STEP 1 and STEP 2, the new $\left(U^{R}, V^{R}\right)$ will satisfy (5.34) and (5.35). However, the jump conditions are not satisfied and the error of which is bounded by

$$
\left|\left(\bar{U}^{R}, \bar{V}^{R}\right)\right| \leq C \epsilon\left(\left|\left\{\delta W^{i,-}\right\}\right|+\left|\left\{\delta W^{i,+}\right\}\right|\right) \leq \frac{1}{2}\left(\left|\left\{\delta W^{i,-}\right\}\right|+\left|\left\{\delta W^{i,+}\right\}\right|\right),
$$

if $\epsilon$ is sufficiently small.

The process of calculating $\left(U^{R}, V^{R}, U^{i}, V^{i}, \lambda, c^{i}\right)$ from jump conditions, that is the three steps as in the proof of this lemma, can be repeated infinitely many times, each time reducing the jump error by at least $1 / 2$. The limit of the iteration process is a true solution of system of this lemma.

Proof of Proposition 5.1. The proof can be obtained by combining the results of Lemmas 5.3 and 5.6. 
Theorem 5.7. Assume that Hypothesis (H1) is satisfied, or equivalently, $\lambda_{0}$ is a simple root for the SLEP function $p(\lambda)$ (Lemma 4.7), there exist unique eigenvalue and eigenfunctions near the approximation $\left(\lambda_{a p}, U_{a p}^{R},\left\{U_{a p}^{i}\right\}\right)$.

Proof. Let the solution mapping of Proposition 5.1 be

$$
\left(U^{R}, V^{R},\left\{U^{i}\right\},\left\{V^{i}\right\}, \lambda,\left\{c^{i}\right\}\right)=\mathcal{F}\left(\left\{\delta W^{i,+}\right\},\left\{\delta W^{i,-}\right\}, h^{R},\left\{h^{i}\right\}\right) .
$$

Then the eigenvalue problem (5.2), (5.3), (5.4), (5.5) can be expressed as

$$
\begin{aligned}
\left(U^{R}, V^{R},\left\{U^{i}\right\},\left\{V^{i}\right\}, \lambda,\left\{c^{i}\right\}\right) \\
\quad=\mathcal{F}\left(\left\{\delta W^{i,+}\right\},\left\{\delta W^{i,-}\right\}, h^{R}+N^{R}\left(U^{R}, \lambda, \epsilon\right),\left\{h^{i}+N^{i}\left(U^{i}, \lambda, c^{i}, \epsilon\right)\right\}\right) .
\end{aligned}
$$

Using the estimates on $N^{R}\left(U^{R}, \lambda, \epsilon\right), N^{i}\left(U^{i}, \lambda, c^{i}, \epsilon\right)$ and Proposition 5.1, we find if $1 / 2<\beta<1$, then $\epsilon^{2 \beta-1}<<1$. Equation (5.44) can be solved by the contraction mapping principle to obtain a unique solution.

Finally, it is easy to verify from the contraction mapping that

$$
\begin{aligned}
& \|\left(U^{R}, V^{R},\left\{U^{i}\right\}\right.\left.,\left\{V^{i}\right\}, \lambda,\left\{c^{i}\right\}\right)\left\|\leq C\left(\|\left\{\delta W^{i,+}\right\},\left\{\delta W^{i,-}\right\}, h^{R},\left\{h^{i}\right\}\right)\right\| \\
& \leq C\left(\epsilon^{m}+\epsilon^{\beta(m+1)-1}+e^{-\alpha \epsilon^{\beta-1}}\left(\epsilon^{\beta-1}\right)^{m} \leq C \epsilon^{\beta(m+1)-1} .\right.
\end{aligned}
$$

As $\epsilon \rightarrow 0,\left(U^{R}, V^{R},\left\{U^{i}\right\},\left\{V^{i}\right\}, \lambda,\left\{c^{i}\right\}\right) \rightarrow 0$. The exact eigenvalue and eigenfunctions are asymptotically $O\left(\epsilon^{\beta(m-1)+2 \beta-1}\right)$ near the approximations $\lambda_{a p}, U_{a p}^{R}$ and $\left\{U_{a p}^{i}\right\}$.

\section{REFERENCES}

[1] Sylvie Benzoni-Gavage, Denis Serre, Kevin Zumbrun, Alternate Evans functions and viscous shock waves. SIAM J. Math. Anal. 32 (2001), no. 5, 929-962.

[2] A. Bressan and R. Colombo, Unique solution of $2 \times 2$ conservation laws with large data, Indiana Univ. Math. J. 44 (1995), 677-725.

[3] A variational calculus for discontinuous solutions of system of conservation laws, Comm. Partial Differential Equations 20 (1995), 1491-1552.

[4] S.-N. Chow, X.-B. Lin and K. Palmer, A shadowing lemma with applications to semilinear parabolic equations, SIAM J. Math. Anal. 20 (1989), 547-557.

[5] W. A. Copple, Dichotomies in stability theory, Lecture Notes in Mathematics, 629, Springer, 1978.

[6] C. M. Dafermos, Solution of the Riemann problem for a class of hyperbolic systems of conservation laws by the viscosity method, Arch. Ration. Mech. Anal. 52 (1973), 1-9.

[7] J. Evans, Nerve axon equations: IV The stable and unstable impulse, Indiana Univ. math. J. 24 (1975), 1169-1190.

[8] A. Freidman, Partial differential equations, Holt. Reinhart and Winston, New York, 1969.

[9] J. K. Hale and X.-B. Lin, Multiple internal layer solutions generated by spatially oscillatory perturbations, J. Differential Equations 154 (1999), 364-418.

[10] A. van Harten, Nonlinear singular perturbation problems: proofs of correctness of a formal approximation based on a contraction principle in a Banach space, J. Math. Anal. Appl., 65(1978), $169-183$.

[11] D. Henry, Geometric theory of semilinear parabolic equations, Lecture Notes in Math. 840, Springer-Verlag, 1981.

[12] C. K. R. T. Jones, Geometric singular perturbation theory, Lecture Notes in Math. 1609, Springer-Verlag, Berlin, 1995, 44-118. 
[13] P. Kokotovic, H. Khalil and J. O'Reilly, Singular perturbation methods in control, Classics in Applied mathematics, SIAM, 1999.

[14] M. Lewicka, Stability conditions for patterns of noninteracting large shock waves, SIAM J. Math. Anal. 32 (2001), 1094-1116.

[15] M. Lewicka, A note on the spectral stability conditions for shock patterns, preprint.

[16] X.-B. Lin, Construction and asymptotic stability of structurally stable internal layer solutions, Trans. Amer. Math. Soc. 353 (2001), 2983-3043.

[17] X.-B. Lin, Analytic semigroup generated by the linearization of a Riemann-Dafermos solution, Dynamics of Partial Differential Equations, 1 (2004) 193-208.

[18] X.-B. Lin, L2 semigroup and linear stability for Riemann solutions of conservation laws, preprint, 2005.

[19] X.-B. Lin and S. Schecter, Stability of self-similar solutions of the Dafermos regularization of a system of conservation laws, SIAM J. Math. Anal. 35 (2003), 884-921.

[20] T. P. Liu, Nonlinear Stability of shock waves for viscous conservation laws, Mem. Amer. math. Soc. 56 (1985), 1-108.

[21] R. E. O'Malley, Introduction to singular perturbations, Academic Press, New York, 1974.

[22] W. Liu, Multiple viscous wave fan profiles for Riemann solutions of hyperbolic systems of conservation laws, Discrete and Continuous Dynamics, 10 (2004), 871-884.

[23] Exponential dichotomies and transversal homoclinic points, J. Differential Equations 55 (1984), 225-256.

[24] A. Pazy, semigroup of linear operators and applications to partial differential equations, Springer, New York, 1983.

[25] Y. Nishiura and H. Fujii, SLEP method to the stability of singularly perturbed solutions with multiple transition layers in reaction-diffusion systems, in Dynamics of Infinite-Dimensional Systems (Lisbon, 1986), 211-230, NATO Adv. Sci. Inst. Ser. F Comput. Systems Sci. 37, Springer, Berlin, 1987.

[26] R. J. Sacker, The splitting index for linear differential systems, J. Differential Equations 33 (1979), 368-405.

[27] R. J. Sacker and G. R. Sell, Existence of dichotomies and invariant splittings for linear differential systems, II, III, J. Differential Equations 22 (1976), 478-496, 497-522.

[28] S. Schecter, Undercompressive shock waves and the Dafermos regularization, Nonlinearity 15 (2002), 1361-1377.

[29] S. Schecter, Computation of Riemann solutions using the Dafermos regularization and continuation, Discrete and Continuous Dynamics, 10 (2004), 965-986.

[30] S. Schecter, Eigenvalue of self-similar solutions of the Dafermos regularization of a system of conservation laws via geometric singular perturbation theory, preprint, 2005.

[31] S. Schochet, Sufficient conditions for local existence via Glimn's scheme for large B.V. data, J. Differential Equations, 89 (1991), 317-354.

[32] D. Serre and K. Zumbrun, Boundary layer stability in real vanishing viscosity limit, Comm. Math. Phys. 221 (2001), 267-292.

[33] H. Suzuki, Y. Nishiura, and H. Ikeda, Stability of traveling waves and a relation between the Evans function and the SLEP method, J. Reine Angew. Math. 475 (1996), 1-37.

[34] P. Szmolyan, in preparation.

[35] V. A. Tupčiev, On the splitting of an arbitrary discontinuity for a system of two first-order quasi-linear equations, USSR Comput. Math. Phys., 4(1964), 36-48.

[36] V. A. Tupčiev, The method of introducing a viscosity in the study of a problem of decay of a discontinuity, Soviet Math. Dokl., 14 (1973), 978-982.

[37] A. E. Tzavaras, Wave interactions and variation estimates for self- similar zero-viscosity limits in systems of conservation laws, Arch. Ration. Mech. Anal. 135 (1996), 1-60.

[38] R. Gardner and K. Zumbrun, The gap lemma and geometric criteria for instability of viscous shock profiles, Comm. Pure Appl. Math. 51 (1998) 797-855. 
[39] K. Zumbrun and P. Howard, Pointwise semigroup methods and stability of viscous shock waves, Indiana Univ. Math. J. 47 (1998), 741-871.

Department of Mathematics, North Carolina State University, Raleigh, NC 276958205 